\title{
Multisensor Fusion Method Based on the Belief Entropy and DS Evidence Theory
}

\author{
Xiaojing Fan $\left(\mathbb{D},{ }^{1,2}\right.$ Yinjing Guo $\mathbb{D}^{1,3}$ Yuanyuan Ju $\mathbb{D},{ }^{1}$ Jiankang Bao $\mathbb{D}^{1},{ }^{1}$ and Wenhong Lyu $\mathbb{D}^{4}$ \\ ${ }^{1}$ College of Electronic and Information Engineering, Shandong University of Science and Technology, Qingdao 266590, China \\ ${ }^{2}$ School of Computer Engineering, Weifang University, Weifang 261061, China \\ ${ }^{3}$ Qingdao Intelligence Ocean Technology Co. Ltd., Qingdao 266590, China \\ ${ }^{4}$ College of Transportation, Shandong University of Science and Technology, Qingdao 266590, China
}

Correspondence should be addressed to Yinjing Guo; gyjlwh@163.com

Received 16 November 2019; Accepted 19 December 2019; Published 16 January 2020

Academic Editor: Xavier Vilanova

Copyright (c) 2020 Xiaojing Fan et al. This is an open access article distributed under the Creative Commons Attribution License, which permits unrestricted use, distribution, and reproduction in any medium, provided the original work is properly cited.

\begin{abstract}
The Dempster-Shafer evidence theory has been widely applied in multisensor information fusion. Nevertheless, illogical results may occur when fusing highly conflicting evidence. To solve this problem, a new method of the grouping of evidence is proposed in this paper. This method uses a combination of the belief entropy and the degree of conflict of the evidence as the judgment rule and divides the entire body of evidence into two separate groups. For the grouped evidence, both the credibility weighted factor based on the belief entropy function and the support weighted factor based on the Jousselme distance function are taken into consideration. The two determined weighted factors are integrated to adjust the evidence before applying the DS combination rule. Numerical examples are provided to demonstrate the theoretical feasibility and rationality of the proposed method. The fusion results indicate that the proposed method is more accurate than the compared algorithms in handling the paradoxes. A decision-making case analysis of the biological system is performed to validate the practical applicability of the proposed method. The results confirm that the proposed method has the highest belief degree of the target concentration $(50.98 \%)$ and has superior accuracy compared to other related methods.
\end{abstract}

\section{Introduction}

Advances in technology have led to difficulties with the use of a single sensor to meet the requirements of information diversification. For this reason, intelligent multisensor systems have attracted the attention of researchers [1-3]. Multisensor systems can be used to obtain different types of information, such as sound and images. So information fusion technology plays a significant role in practical applications of intelligent multisensor systems [4-7]. Multisensor information fusion technology [8] deals with the independent observation data that are obtained from multiple sensors by selecting the appropriate information-processing algorithm. It can make complete and accurate decisions on targets in a variety of complex, dynamic, and uncertain environments. It can eliminate the limitations of single sensors that can only obtain partial information on a target. The traditional multisensor fusion methods include Kalman filtering
[9], the statistical method [10], Bayesian inference [11], empirical reasoning [12], the template method [12], and others $[13,14]$. In practical applications, the data that are acquired from a multisensor system may be incomplete or inaccurate due to the internal and external effects [15] of bad weather, a sensor fault, an insufficient energy supply, a communication failure, etc. These unreliable data may lead to the information having a certain degree of uncertainty and fuzziness, resulting in contradictory fusion results [16, 17]. A number of intelligent algorithms have been applied in recent years to solve this issue, including the adaptive neural network [18], the rough set theory $[19,20]$, the fuzzy set theory [21-23], the evidence theory [24, 25], and others [26-29].

The Dempster-Shafer evidence theory $[24,25]$, which has a rigorous theoretical foundation and simple combination rules, is widely applied in various fields of information fusion, including decision-making [30-33], target recognition [34-36], risk analysis [37, 38], human reliability 
analysis [39], supplier selection [40], aphasia diagnosis [41], fault diagnosis [42-44], and others [45, 46]. However, when fusing high conflict evidence, the DS evidence theory may generate illogical results, which limits its practical application [47]. Over the last few decades, research on conflicting evidence has become a mainstream trend. The existing methods can be divided into two main types.

The first type of algorithm focuses on the modification of the normalization step of the DS combination rule. Smets proposed a transferable confidence rule [48] that assigns the conflicting sections to empty sets. Dubois and Prade proposed a disjunctive combination rule [49] in which the reliability of the conflicting evidence is redistributed to other evidences. Yager proposed a nonregularization rule [50] that treats the conflicting evidence as unreliable information and assigns the conflicting section to $\Theta$. Sun et al. proposed the conflict average rule [51] which turns the combination rule into an operation that calculates an average. Furthermore, these methods may destroy the commutativity and the associativity of the combination rule and increase the number of required computations.

The second type of algorithm focuses on preprocessing the original evidence. Algorithms of this type include Murphy [52], Han et al. [53], Zhang et al. [54], Yuan et al. [55], Xiao [15], Radim and Prakash [56], Ye et al. [57], Khan and Anwar [58], and Ma and An and Jiang et al. [59, 60]. Murphy [52] averaged multiple sets of evidence without considering the correlation between the sets of evidence. Han et al. [53] used the Jousselme distance function [61, 62] to obtain the distance between two groups of evidence to calculate the similarity between the groups of evidence. The support degree and the credibility degree of the evidence were obtained based on a similarity measurement matrix. The modified average (or the weight average) of the evidence can be used to obtain the result where the credibility degree was a weight. Zhang et al. [54] introduced the concept of a vector space and used the cosine function to deal with conflicting evidence. Yuan et al. [55] proposed a novel weighted evidence combination rule. The Jousselme distance function and the belief entropy were combined to obtain the dynamic reliability of each sensor. A weighted averaging method was adopted to modify the conflict evidence by assigning different weights to evidence according to sensor reliability. Xiao [15] introduced the concept of belief entropy to express the effect of the evidence itself based on Deng entropy [63] and integrated the Belief Jensen-Shannon divergence with the belief entropy as the final weight for each piece of evidence. Radim and Prakash [56] proposed a new definition of entropy of BPA in the DS theory based on Shannon entropy [64]. The method defined the sum of an expected value of Shannon's entropy as a measure of conflict and the expected value of Hartley's entropy as a measure of nonspecificity. Ye et al. [57] proposed a robust DS combination method based on evidence correction and conflict redistribution. The Matusita distance function and closeness degree function were combined to modify the evidence. Based on the weighted mass function, they designed rational mass assignment of conflicting probability instead of directly employing the DS combination rule. Khan and Anwar [58] proposed a novel entropy function based on Shannon entropy, which was better at capturing uncertainties compared to Shannon and Deng entropies. Meanwhile, an 8-step algorithm had been developed which can eliminate the inherent paradoxes of a classical DS theory. These methods can solve the problem of illogical results of evidence fusion to some extent and affirmed the validity of the Jousselme distance function and the information entropy function. Based on previous studies, we are still persistent to achieve more accurate fusing results.

In this paper, we propose a new method of the grouping of evidence. The main contributions of this paper can be summarized as follows: First, the proposed method improves the accuracy of evidence fusion by considering both the evidence distance function and the information volume. Second, this method significantly reduces the calculation burden through the grouping of evidence. We use a combination of belief entropy and the degree of conflict of the evidence as the judgment rule and divide the entire body of evidence into two separate categories: high credibility degree evidence and general credibility degree evidence. During the processing of the grouped evidence, both the credibility degree and the support degree are taken into consideration. The proposed method assigns a more appropriately weighted factor to every piece of evidence before applying the DS combination rule. Firstly, the credibility degree of the evidence itself is measured by taking advantage of the belief entropy function. The credibility weighted factor can then be determined appropriately. Secondly, the support weighted factor of the evidence is determined by making use of the Jousselme distance function. Finally, both the credibility weighted factor and the support weighted factor are integrated to form the final weight to adjust the bodies of evidence before applying the DS combination rule. Numerical examples of various paradoxes and a case analysis of the target decision-making on a biological system are presented to demonstrate the feasibility and rationality of the proposed method.

This paper is organized as follows: The DS evidence theory, the belief entropy, and the Jousselme distance function are briefly reviewed in Section 2. The implementation model, specific steps, and the flowchart of the proposed method based on the belief entropy and the information support degree are expounded in Section 3. Numerical examples of various paradoxes in extreme cases are provided to demonstrate the theoretical feasibility and rationality of the proposed method in Section 4. A case analysis of the target concentration decision-making in a hydroponic system is provided to establish the effectiveness of the proposed method in a practical application in Section 5. Finally, Section 6 presents the conclusions and forecasts.

\section{Theoretical Foundations}

2.1. DS Evidence Theory. The DS evidence theory $[24,25]$ is, in a way, a generalization of the probability theory and Bayesian reasoning. Based on the accretion of evidence, the DS evidence theory enables a multisensor system to provide valid and accurate information fusion results without the requirement of prior information and conditional probabilities. The 
DS evidence theory is capable of effectively handling system uncertainties [17]. The basic concepts are described below.

Definition 1. Define $\Theta$ as the frame of discernment (FOD), which consists of $N$ mutually exclusive and collectively exhaustive hypotheses. FOD is defined as the form of the function set as follows:

$$
\Theta=\left\{\theta_{1}, \theta_{2}, \cdots, \theta_{N}\right\}=\left\{\theta_{i} \mid i=1,2, \cdots, N\right\},
$$

where $N$ is the number of hypotheses in the system and $\theta_{i}$ is an element of the FOD.

Define $2^{\Theta}$ as the power set based on the FOD, which is composed of $2^{N}$ propositions of $\Theta$.

$2^{\Theta}=\left\{\varnothing,\left\{\theta_{1}\right\},\left\{\theta_{2}\right\}, \cdots,\left\{\theta_{N}\right\},\left\{\theta_{1}, \theta_{2}\right\},\left\{\theta_{1}, \theta_{3}\right\}, \cdots,\left\{\theta_{1}, \theta_{N}\right\}, \cdots, \Theta\right\}$.

Definition 2. Define $m(A)$ as the mass function or the basic probability assignment (BPA) that satisfies the following condition. $A$ represents any proposition of $2^{\Theta}$.

$$
\left\{\begin{array}{l}
\sum_{A \subseteq 2^{\Theta}} m(A)=1, \\
m(\varnothing)=0 .
\end{array}\right.
$$

The proposition $A$ that satisfies $m(A)>0$ is known as the focal element.

Define vector $\mathbf{m}_{i}$ to represent the evidence $\mathbf{m}_{i}=\left[m_{i}\left(A_{1}\right)\right.$, $\left.m_{i}\left(A_{2}\right), \cdots, m_{i}\left(A_{m}\right)\right](i=1,2, \cdots, n)$ for convenience. $\mathscr{F}=\left\{A_{1}\right.$, $\left.A_{2}, \cdots, A_{m}\right\}$ is the set of all the focal elements included in the evidence $\mathbf{m}_{i}, A_{1}, A_{2}, \cdots, A_{m} \subseteq 2^{\Theta}$. Each of $A_{k}(k=1,2$, $\cdots, m)$ has a fixed value (BPA) $m\left(A_{k}\right)$.

Definition 3. Define $\mathbf{m}_{1}, \mathbf{m}_{2}$ as two sources of evidence on the frame of discernment $\Theta$. The DS combination rule is defined as under.

$$
\begin{aligned}
& \text { If and only if } K=\sum_{A_{i} \cap B_{j}=\varnothing} m_{1}\left(A_{i}\right) m_{2}\left(B_{j}\right)<1 \text {. } \\
& \qquad \begin{array}{c}
\mathbf{m}(A)=\frac{1}{1-K} \sum_{A_{i} \cap B_{j}=A} m_{1}\left(A_{i}\right) m_{2}\left(B_{j}\right), \quad A \neq \varnothing, \\
K=\sum_{A_{i} \cap B_{j}=\varnothing} m_{1}\left(A_{i}\right) m_{2}\left(B_{j}\right) .
\end{array}
\end{aligned}
$$

$K$ is a conflicting factor that reflects the degree of conflict between pieces of evidence.

2.2. Information Entropy. Shannon [64, 65] introduced information entropy to solve the problem of the quantitative measurement of information. Information entropy does not require a specific condition of the probability distribution.

Deng [63] generalized Shannon entropy and proposed a generalized method to measure uncertain information. The main concepts are defined below.
Definition 4. Define $\mathbf{m}_{i}=\left[m_{i}\left(A_{1}\right), m_{i}\left(A_{2}\right), \cdots, m_{i}\left(A_{m}\right)\right]$, which satisfies $\sum_{k=1}^{m} m_{i}\left(A_{k}\right)=1$ as the $i$ th $(i=1,2, \cdots, n)$ piece of evidence generated from the information source. Then, the information entropy (Deng entropy) of the $i$ th piece of evidence $\mathbf{m}_{i}$ shall be derived:

$$
E_{d}\left(\mathbf{m}_{i}\right)=-\sum_{k=1}^{m} m_{i}\left(A_{k}\right) \log \frac{m_{i}\left(A_{k}\right)}{2^{\left|A_{k}\right|}-1} .
$$

$A_{k} \subseteq \mathscr{F},\left|A_{k}\right|$ is the cardinality of $A_{k}$.

When $A_{k}(k=1,2, \cdots, m)$ is the single-element focal element, Deng entropy degenerates to Shannon entropy, namely,

$$
\begin{aligned}
E_{d}\left(\mathbf{m}_{i}\right) & =-\sum_{k=1}^{m} m_{i}\left(A_{k}\right) \log \frac{m_{i}\left(A_{k}\right)}{2^{\left|A_{k}\right|}-1} \\
& =-\sum_{k=1}^{m} m_{i}\left(A_{k}\right) \log m_{i}\left(A_{k}\right) .
\end{aligned}
$$

The Deng entropy has proved to be an efficient method to measure the degree of uncertainty in the evidence that is expressed not only by the probability distribution but also by the basic probability assignment (BPA). The greater the Deng entropy of the evidence, the greater shall the degree of uncertainty be, and the credibility degree of the evidence shall be lower. Evidence that has a high credibility degree can indeed play a crucial role in the final combination.

2.3. Jousselme Distance Function. Jousselme et al. [61, 62] introduced a measure of performance (MOP) for identification algorithms based on the DS evidence theory. A principled distance between the sets of BPAs based on a quantification of the similarity was first proposed and indicated that the proposed distance meets the requirement for the metric.

Definition 5. Let $\mathbf{m}_{i}$ and $\mathbf{m}_{j}$ be the two sources of evidence in the frame of discernment $\Theta$. The Jousselme distance between $\mathbf{m}_{i}$ and $\mathbf{m}_{j}$ is

$$
d\left(\mathbf{m}_{i}, \mathbf{m}_{j}\right)=\sqrt{\frac{1}{2}\left(\mathbf{m}_{i}-\mathbf{m}_{j}\right)^{\mathrm{T}} \mathbf{D}\left(\mathbf{m}_{i}-\mathbf{m}_{j}\right)},
$$

where $\mathbf{D}=\left[D\left(\mathbf{m}_{i}, \mathbf{m}_{j}\right)=\left(\left|A_{s} \cap B_{t}\right|\right) /\left(\left|A_{s} \cup B_{t}\right|\right)\right], A_{s}, B_{t} \subset 2^{\Theta}$.

Equation (9) can be obtained after simplifying Equation (8).

$$
\begin{aligned}
d\left(\mathbf{m}_{i}, \mathbf{m}_{j}\right) & =\sqrt{\frac{1}{2}\left(\left\|\mathbf{m}_{i}\right\|^{2}+\left\|\mathbf{m}_{j}\right\|^{2}-2\left\langle\mathbf{m}_{i}, \mathbf{m}_{j}\right\rangle\right)}, \\
\left\langle\mathbf{m}_{i}, \mathbf{m}_{j}\right\rangle & =\sum_{s=1}^{2^{N}} \sum_{t=1}^{2^{N}} m_{i}\left(A_{s}\right) m_{j}\left(B_{t}\right) \frac{\left|A_{s} \cap B_{t}\right|}{\left|A_{s} \cup B_{t}\right|}
\end{aligned}
$$

\section{Materials and Methods}

The credibility degree of evidence obtained through data collection and modeling has been found not to be exactly 


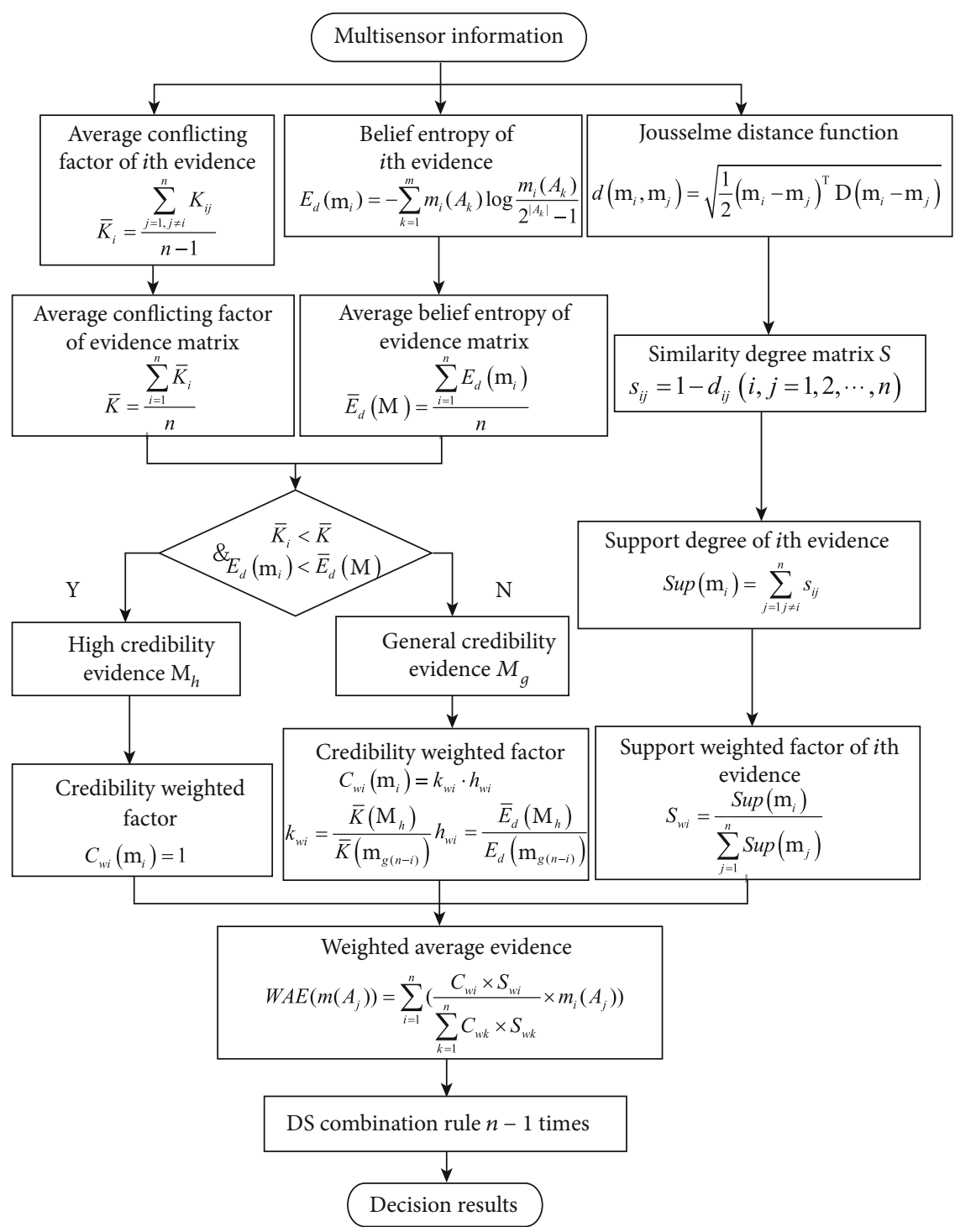

FIGURE 1: Implementation block of the proposed method.

equal. Hence, it is not able to adequately represent the comprehensive weight of the evidence; rather, it only considers the similarity degree of the evidence. In this paper, a new multisensor information fusion method is presented. The proposed method is summarized in two main procedures: one is the grouping of evidence and another is the weighting revising of the grouped evidence. Specific steps are as follows: First, the evidence is divided into two separate categories according to the judgment rule. Second, the credibility weighted factors are calculated based on the belief entropy. The type of evidence with a high credibility degree is allocated a constant weight. The other type of evidence is allocated a corresponding weight. Third, the support weighted factors of the evidence are calculated by making use of the Jousselme distance function. Finally, the two corrected factors are integrated to form the final weight to adjust the bodies of the evidence before using the DS combination rule. Figure 1 shows the flowchart of the proposed method.

\subsection{Calculate the Credibility Weighted Factor of the Evidence}

Step 1. There are $n$ pieces of evidence. Establish the evidence matrix $\mathbf{M} . \quad \mathbf{M}=\left[\mathbf{m}_{1} \mathbf{m}_{2} \cdots \mathbf{m}_{n}\right]^{\mathrm{T}}$. The conflicting factor between $\mathbf{m}_{i}(i=1,2, \cdots, n)$ and $\mathbf{m}_{j}(j=1,2, \cdots, n)$ is calculated via Equation (5). The conflicting factor matrix $\mathbf{K}$ can be constructed as follows:

$$
\mathbf{K}=\left[\begin{array}{cccc}
K_{11} & K_{12} & \cdots & K_{1 n} \\
K_{21} & K_{22} & \cdots & K_{2 n} \\
\vdots & \vdots & \ddots & \vdots \\
K_{n 1} & K_{n 2} & \cdots & K_{n n}
\end{array}\right] .
$$

Step 2. The average conflicting factor $\bar{K}_{i}$ of the evidence $\mathbf{m}_{i}$ $(i=1,2, \cdots, n)$ is calculated. 


$$
\bar{K}_{i}=\frac{\sum_{j=1, j \neq i}^{n} K_{i j}}{n-1} .
$$

The average conflicting factor $\bar{K}$ of the conflicting factor matrix $\mathbf{K}$ is calculated.

$$
\bar{K}=\frac{\sum_{i=1}^{n} \bar{K}_{i}}{n} .
$$

Step 3. By making use of the belief entropy function (Equation (6)), the belief entropy of $\mathbf{m}_{i}(i=1,2, \cdots, n)$, denoted as $E_{d}\left(\mathbf{m}_{i}\right)$, can be obtained. The belief entropy matrix $E_{d}(\mathbf{M})$ can be constructed as follows:

$$
E_{d}(\mathbf{M})=\left[\begin{array}{llll}
E_{d}\left(\mathbf{m}_{1}\right) & E_{d}\left(\mathbf{m}_{2}\right) & \cdots & E_{d}\left(\mathbf{m}_{n}\right)
\end{array}\right]^{\mathrm{T}} .
$$

Step 4 . The average belief entropy $\bar{E}_{d}(\mathbf{M})$ is calculated.

$$
\bar{E}_{d}(\mathbf{M})=\frac{\sum_{i=1}^{n} E_{d}\left(\mathbf{m}_{i}\right)}{n} .
$$

Step 5. The evidence is divided into two categories based on the following rules. One category is the high credibility degree matrix, denoted $\mathbf{M}_{h}, \mathbf{M}_{h}=\left[\begin{array}{lllll}\mathbf{m}_{h 1} & \mathbf{m}_{h 2} & \cdots & \mathbf{m}_{h i}\end{array}\right]^{\mathrm{T}}$ $(i \leq n)$, which must satisfy $\bar{K}_{i}<\bar{K}$ and $E_{d}\left(\mathbf{m}_{i}\right)<\bar{E}_{d}(\mathbf{M})$. The other category is the general credibility degree matrix, denoted $\mathbf{M}_{g}, \mathbf{M}_{g}=\left[\begin{array}{llll}\mathbf{m}_{g 1} & \mathbf{m}_{g 2} & \cdots & \mathbf{m}_{g(n-i)}\end{array}\right]^{\mathrm{T}}$ which does not need to meet any of the above-described conditions.

Step 6. The evidence in the high credibility degree matrix $\mathbf{M}_{h}$ does not need to be corrected; that is, the credibility weighted factor defaults to 1, i.e., $C_{w i}\left(\mathbf{m}_{i}\right)=1$.

The evidence in the general credibility degree matrix $\mathbf{M}_{g}$ should be corrected by two factors. The credibility weighted factor $C_{w i}\left(\mathbf{m}_{i}\right)$ can be obtained as follows:

$$
C_{w i}\left(\mathbf{m}_{i}\right)=k_{w i} \cdot h_{w i}
$$

We consider two attributes of evidence, the conflicting attribute between evidences and the belief entropy attribute of evidence itself. The combination of subjective and objective attributes can make the measurement to comprehensive credibility of evidence more accurate and the grouping of evidence more reasonable. The credibility weighted factor $C_{w i}\left(\mathbf{m}_{i}\right)$ is the proportionality coefficient of comprehensive credibility of evidence, which is equal to the product of the credibility of two attributes. For the high credibility degree evidence, we take normalization constant as the credibility of all the attributes, that is, $C_{w i}\left(\mathbf{m}_{i}\right)=1 \times 1=1$. For the general credibility degree evidence, $C_{w i}\left(\mathbf{m}_{i}\right)$ is equal to the product of the transfer factor of the attribute credibility (Equation (15)). The transfer factor is the corresponding ratio of the credibility while transforming the general credibility degree evidence into the high credibility degree evidence. The numerical computation is accomplished using the data of the high credibility degree evidence as the reference value. $k_{w i}$ is the conflicting transfer factor, which satisfies the permutation identityl $\cdot \bar{K}\left(\mathbf{M}_{h}\right)=k_{w i} \cdot \bar{K}_{i}\left(\mathbf{m}_{g(n-i)}\right)$. So we can obtain $k_{w i}=\left(\bar{K}\left(\mathbf{M}_{h}\right)\right) /\left(\bar{K}_{i}\left(\mathbf{m}_{g(n-i)}\right)\right) . \bar{K}\left(\mathbf{M}_{h}\right)$ is the average conflicting factor of the high credibility degree matrix $\mathbf{M}_{h}$ derived by Equation (12). $\bar{K}_{i}\left(\mathbf{m}_{g(n-i)}\right)$ is the average conflicting factor of the general credibility degree evidence $\mathbf{m}_{g(n-i)}$ derived by Equation (11).

$h_{w i}$ is the belief entropy transfer factor, which satisfies the permutation identityl $\cdot \bar{E}_{d}\left(\mathbf{M}_{h}\right)=h_{w i} \cdot E_{d}\left(\mathbf{m}_{g(n-i)}\right)$. So we can obtain $h_{w i}=\left(\bar{E}_{d}\left(\mathbf{M}_{h}\right)\right) /\left(E_{d}\left(\mathbf{m}_{g(n-i)}\right)\right) . \bar{E}_{d}\left(\mathbf{M}_{h}\right)$ is the average belief entropy of the high credibility degree matrix $\mathbf{M}_{h}$ derived by Equation (14). $E_{d}\left(\mathbf{m}_{g(n-i)}\right)$ is the belief entropy of the general credibility degree evidence $\mathbf{m}_{g(n-i)}$ derived by Equation (6).

The final credibility weighted factor $C_{w i}$ on the basis of Equation (15) after normalization is given below:

$$
C_{w i}=\frac{C_{w i}\left(\mathbf{m}_{i}\right)}{\sum_{j=1}^{n} C_{w j}\left(\mathbf{m}_{j}\right)} .
$$

\subsection{Calculate the Support Weighted Factor of the Evidence}

Step 1. By making use of the Jousselme distance function (Equation (8)), the Jousselme distance between $\mathbf{m}_{i}(i=1,2$, $\cdots, n)$ and $\mathbf{m}_{j}(j=1,2, \cdots, n)$ can be calculated. A distance measure matrix $\mathbf{D}$, namely, the conflict matrix of the evidence, can be constructed as follows:

$$
\mathbf{D}=\left[\begin{array}{cccc}
0 & d_{12} & \cdots & d_{1 n} \\
d_{21} & 0 & \cdots & d_{2 n} \\
\vdots & \vdots & \cdots & \vdots \\
d_{n 1} & d_{n 2} & \cdots & 0
\end{array}\right] .
$$

Step 2. The similarity matrix $\mathbf{S}$ of the evidence is derived.

$$
\mathbf{S}=\mathbf{I}-\mathbf{D}=\left[\begin{array}{cccc}
1 & s_{12} & \cdots & s_{1 n} \\
s_{21} & 1 & \cdots & s_{2 n} \\
\vdots & \vdots & \cdots & \vdots \\
s_{n 1} & s_{n 2} & \cdots & 1
\end{array}\right] .
$$

where $s_{i j}=1-d_{i j}(i, j=1,2, \cdots, n), s_{i j}=s_{j i}$, and $\mathbf{S}$ is a symmetric matrix.

The degree of mutual support shows a gradual increase along with an increase in the similarity between the two pieces of evidence. Conversely, the degree of mutual support shows a gradual decrease along with a decrease in the similarity between the two pieces of evidence.

Step 3. The support degree $\operatorname{Sup}\left(\mathbf{m}_{i}\right)$ of the BPA of the evidence $\mathbf{m}_{i}(i=1,2, \cdots, n)$ is calculated.

$$
\operatorname{Sup}\left(\mathbf{m}_{i}\right)=\sum_{j=1 j \neq i}^{n} s_{i j}(i, j=1,2, \cdots, n) .
$$


The support weighted factor of $\mathbf{m}_{i}(i=1,2, \cdots, n)$ is normalized as below.

$$
S_{w i}=\frac{\operatorname{Sup}\left(\mathbf{m}_{i}\right)}{\sum_{j=1}^{n} \operatorname{Sup}\left(\mathbf{m}_{j}\right)} \quad(i, j=1,2, \cdots, n) .
$$

\subsection{Generate and Fuse the Weighted Evidence}

Step 1. The credibility weighted factor $C_{w i}$ and the support weighted factor $S_{w i}$ are integrated to form the final weight to adjust the bodies of evidence. The weighted average evidence $\operatorname{WAE}\left(m\left(A_{j}\right)\right)$ is obtained as follows:

$\operatorname{WAE}\left(m\left(A_{j}\right)\right)=\sum_{i=1}^{n}\left(\frac{C_{w i} \times S_{w i}}{\sum_{k=1}^{n} C_{w k} \times S_{w k}} \times m_{i}\left(A_{j}\right)\right) \quad(i=1,2, \cdots, n)$.

Step 2. The weighted average evidence $\operatorname{WAE}\left(m\left(A_{j}\right)\right)$ is fused via the DS combination rule (Equation (4)) $n$ - 1 times. Then, the final result of the combination of the evidence from multiple sensors can be obtained.

\section{Numerical Experiments and Analyses}

In this section, with an intent to establish the theoretical feasibility and rationality of the proposed method, we provide various numerical examples of different paradoxes in extreme cases.

Evidently, the value of a logarithm operation has no meaning when the BPA assignment value is zero. Hence, in this pertinent case, a very small number $1 \times 10^{-12}$ was used to replace the zero value, as it has been proven that this would not affect the results of the calculation [66].

4.1. Completely Conflicting Paradox. In the multisensor system, there are three sources of evidence on the frame of discernment $\Theta=\{A, B, C\}$

$$
\begin{aligned}
& E 1: m_{1}(A)=1, m_{1}(B)=0, m_{1}(C)=0, \\
& \text { E2 }: m_{2}(A)=0, m_{2}(B)=1, m_{2}(C)=0, \\
& \text { E3 }: m_{3}(A)=0.9, m_{3}(B)=0.1, m_{3}(C)=0 .
\end{aligned}
$$

The conflicting factor of the first and the second sets of evidence can be computed by Equation (5), $K=1$. However, the DS combination rule cannot be applied because of the denominators that have a value of zero. Although the pieces of evidence $E 1$ and $E 3$ support the proposition $A$, the final synthetic results are completely contrary to the goal. Figure 2(a) and Table 1 show the fusion results of the different methods in the completely conflicting paradox.

4.2. 0 Trust Paradox. In the multisensor system, there are three sources of evidence on the frame of discernment $\Theta=\{A, B, C\}$.

$$
\begin{aligned}
& E 1: m_{1}(A)=0.5, m_{1}(B)=0.2, m_{1}(C)=0.3, \\
& E 2: m_{2}(A)=0, m_{2}(B)=0.9, m_{2}(C)=0.1, \\
& E 3: m_{3}(A)=0.55, m_{3}(B)=0.1, m_{3}(C)=0.35 .
\end{aligned}
$$

The following DS fusion result was obtained by making use of the DS combination rule (Equation (4)): $m(A)=0$, $m(B)=0.6316, \quad m(C)=0.3684, \quad K=0.9715$. The fusion result is found to always be zero for the focal element $A$, since a value of zero is assigned to proposition $A$ in the piece of evidence $E 2$, no matter how large the basic probability assignment that the pieces of evidence $E 1$ and E3 conformably allocate to proposition $A$ and no matter how many pieces of evidence have been waiting for fusion after the piece of evidence E2. The piece of evidence $E 2$ has a one ballot veto for the fusion of the entire body of evidence. Figure 2(b) and Table 1 show the fusion results of the different methods in the 0 trust paradox.

4.3. 1 Trust Paradox. In the multisensor system, there are two sources of evidence on the frame of discernment $\Theta=\{A, B, C\}$.

$$
\begin{aligned}
& E 1: m_{1}(A)=0.9, m_{1}(B)=0.1, m_{1}(C)=0, \\
& E 2: m_{2}(A)=0, m_{2}(B)=0.1, m_{2}(C)=0.9 .
\end{aligned}
$$

The following DS fusion result was obtained by making use of the DS combination rule (Equation (4)): $m(A)=$ $0, m(B)=1, m(C)=0, K=0.9900$. The DS tends to generate illogical results and recognizes the object $B$ as the target proposition. No matter how small the basic probability assignment that the pieces of evidence $E 1$ and $E 2$ conformably allocate to proposition $B$, the fusion result shall always be $m(B)=1$. By virtue of the larger conflict in the evidence, the small probability event becomes the inevitable event through the evidence fusion. Figure 2(c) and Table 1 show the fusion results of the different methods in the 1 trust paradox.

4.4. Highly Conflicting Paradox. In the multisensor system, there are five sources of evidence on the frame of discernment $\Theta=\{A, B, C\}$.

$$
\begin{aligned}
& E 1: m_{1}(A)=0.3, m_{1}(B)=0.2, m_{1}(C)=0.1, m_{1}(\Theta)=0.4, \\
& E 2: m_{2}(A)=0, m_{2}(B)=0.9, m_{2}(C)=0.1, m_{2}(\Theta)=0, \\
& \text { E3: } m_{3}(A)=0.6, m_{3}(B)=0.1, m_{3}(C)=0.1, m_{3}(\Theta)=0.2, \\
& \text { E4: } m_{4}(A)=0.7, m_{4}(B)=0.1, m_{4}(C)=0.1, m_{4}(\Theta)=0.1, \\
& \text { E5: } m_{5}(A)=0.7, m_{5}(B)=0.1, m_{5}(C)=0.1, m_{5}(\Theta)=0.1 .
\end{aligned}
$$

The fusion results and the conflicting factor were acquired through Equations (4) and (5), respectively. $m(A)$ $=0, m(B)=0.9153, m(C)=0.0847, m(\Theta)=0, \quad K=0.8000$. The DS fusion results lend the proposition $C$ a higher belief degree and completely negate the true proposition $A$. 


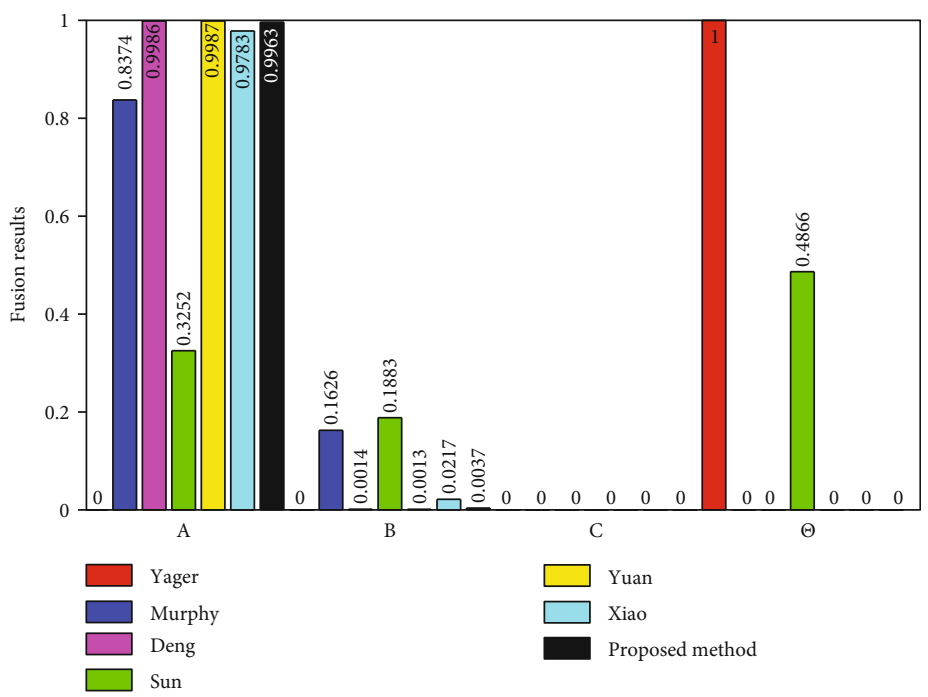

(a)

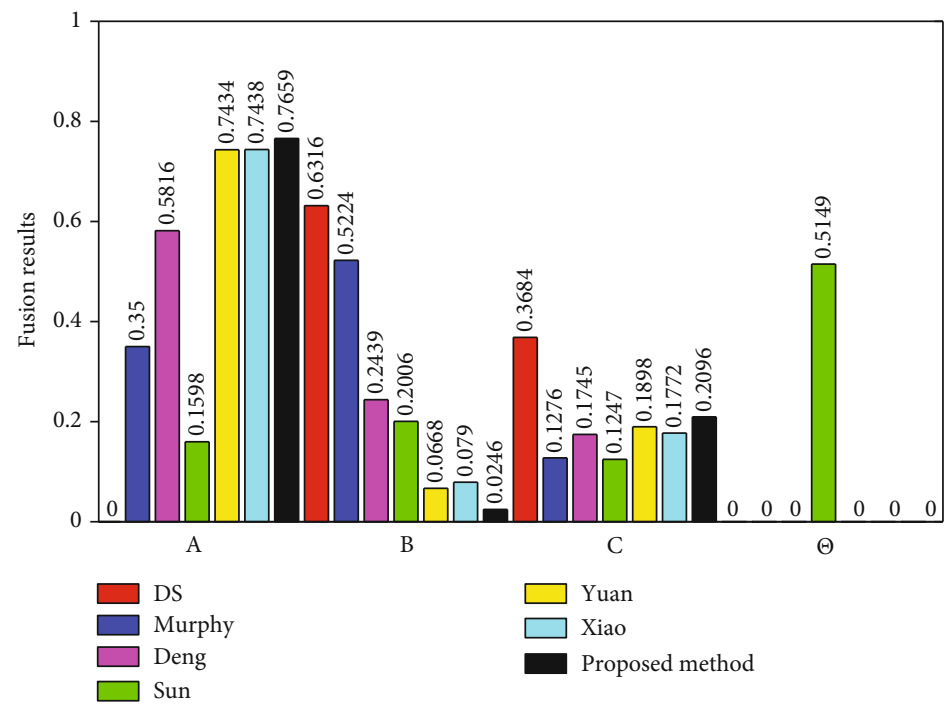

(b)

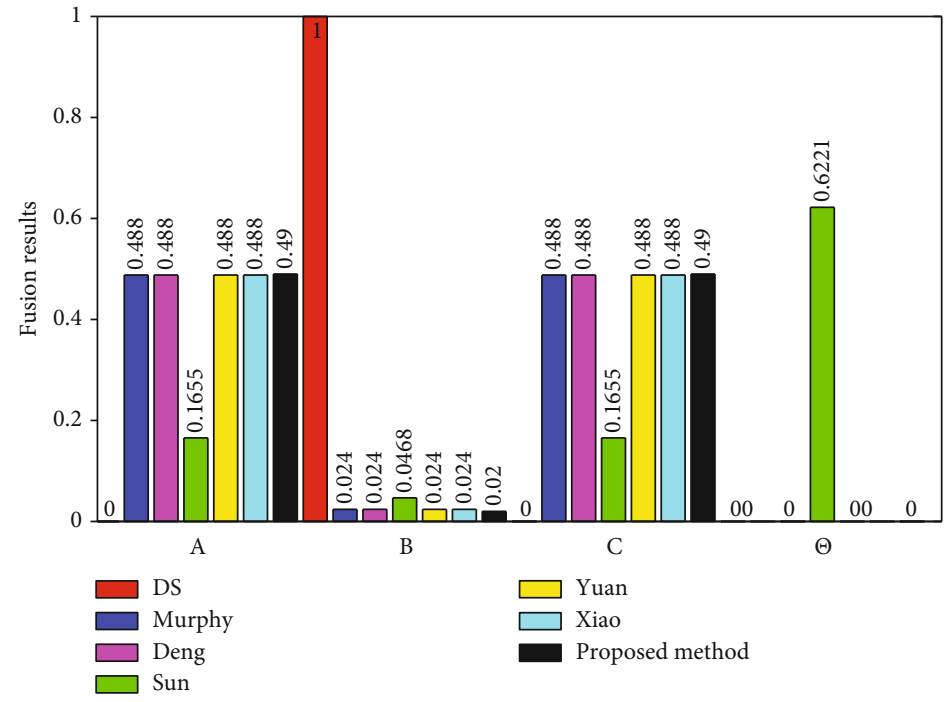

(c)

Figure 2: Continued. 


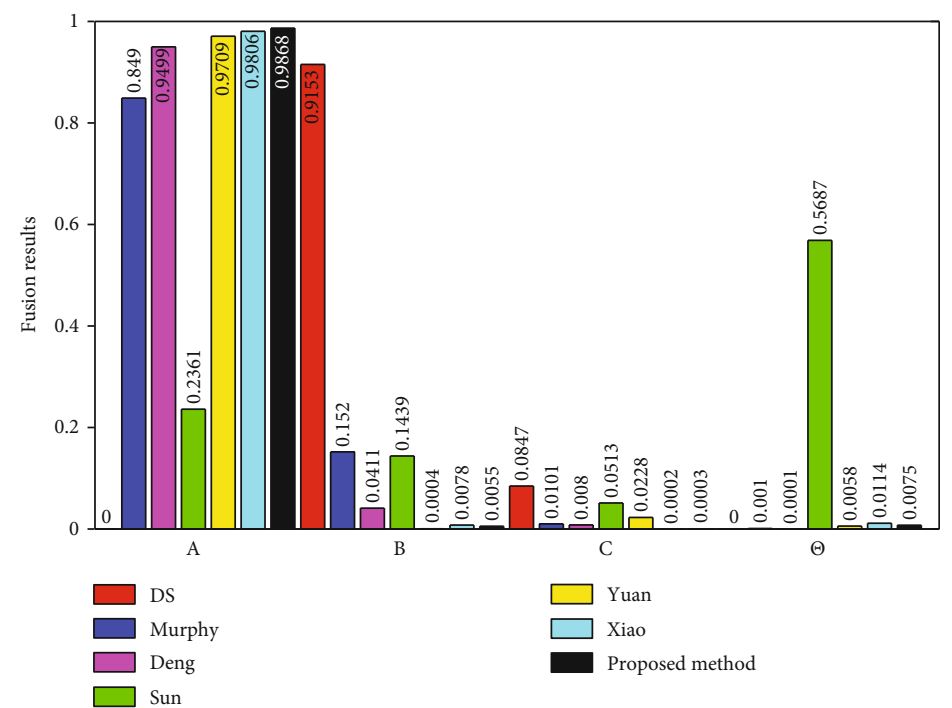

(d)

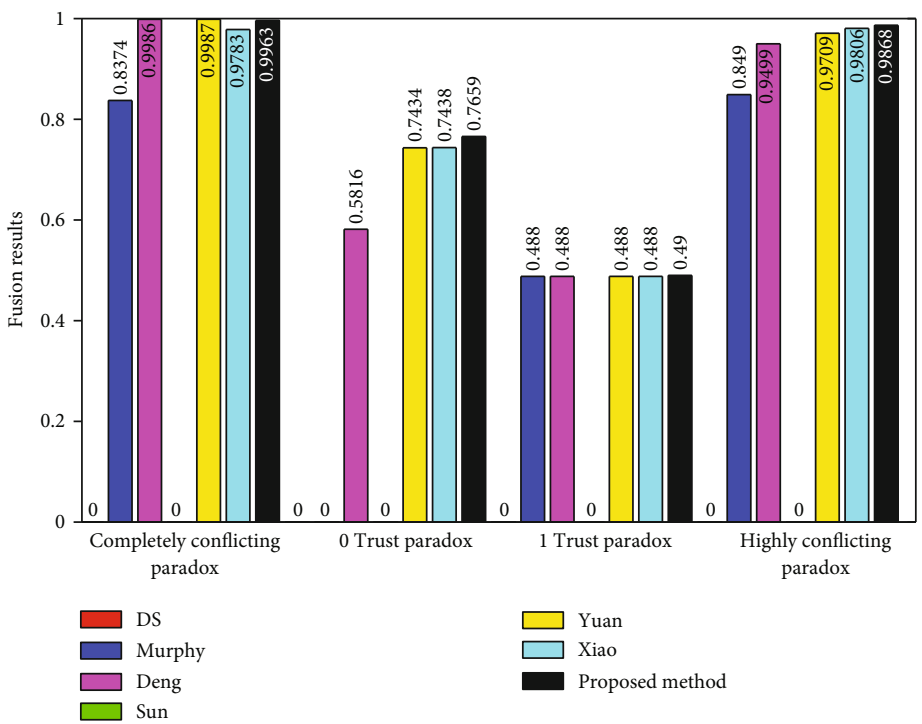

(e)

Figure 2: Fusion results of the different paradoxes. (a) Fusion results in completely conflicting paradox. (b) Fusion results in 0 trust paradox. (c) Fusion results in 1 trust paradox. (d) Fusion results in highly conflicting paradox. (e) Fusion results of the target proposition $A$.

Figure $2(\mathrm{~d})$ and Tables 1 and 2 show the fusion results of the different methods in the highly conflicting paradox.

4.5. Analyses. As shown in Figure 2(a), the DS evidence theory combination rule becomes invalid because of $K=1$ in the complete conflict paradox. Yager and Sun are not able to identify the right target proposition $A$. Yager gives $\Theta$ the belief degree of $m(\Theta)=1$. Sun's method has good combination rules but is only able to solve some of the conflicts, and the BPA for $\Theta$ is still as high as $m(\Theta)=0.4866$. Murphy is able to identify the target proposition $A$ with low precision $(m(A)=0.8374)$. Deng's method, Yuan's method, Xiao's method, and the proposed method are able to obtain the correct conclusion with high precision in the complete conflict paradox.
As shown in Figure 2(b), the DS algorithm lends proposition $B$ the higher belief degree of $m(B)=0.6316$, which alone does not lead to the right decision. Sun gives a BPA for $\Theta$ that is as high as $m(\Theta)=0.5149$, which indicates the uncertainty in the target proposition. Murphy obtains the wrong result about the target proposition being $B$. Deng's method, Yuan's method, Xiao's method, and the proposed method can solve the conflicts in the evidence and obtain the most effective results on the right target proposition $A$ in 0 trust paradox.

In the 1 trust paradox, we selected two sources of evidence with the greatest conflicts as an example. Figure 2(c) shows the simulation results. DS $(m(B)=1)$ and Sun $(m(\Theta)=0.6221)$ choose the wrong target proposition and are not able to resolve the conflicts in this paradox. Murphy's 
TABLE 1: Fusion results of the different paradoxes.

\begin{tabular}{|c|c|c|c|c|c|c|}
\hline \multirow{2}{*}{ Paradoxes } & \multirow{2}{*}{ Methods } & \multicolumn{4}{|c|}{ Propositions } & \multirow{2}{*}{ Target } \\
\hline & & $A$ & $B$ & C & $\Theta$ & \\
\hline \multirow{7}{*}{ Completely conflicting paradox } & Yager [50] & 0 & 0 & 0 & 1 & $\Theta$ \\
\hline & Murphy [52] & 0.8374 & 0.1626 & 0 & 0 & $A$ \\
\hline & Deng [53] & 0.9986 & 0.0014 & 0 & 0 & $A$ \\
\hline & Sun $[51]$ & 0.3252 & 0.1883 & 0 & 0.4866 & $\Theta$ \\
\hline & Yuan [55] & 0.9987 & 0.0013 & 0 & 0 & $A$ \\
\hline & Xiao [15] & 0.9783 & 0.0217 & 0 & 0 & $A$ \\
\hline & Proposed & 0.9963 & 0.0037 & 0 & 0 & $A$ \\
\hline \multirow{7}{*}{0 trust paradox } & DS [24] & 0 & 0.6316 & 0.3684 & 0 & $B$ \\
\hline & Murphy [52] & 0.3500 & 0.5224 & 0.1276 & 0 & $B$ \\
\hline & Deng [53] & 0.5816 & 0.2439 & 0.1745 & 0 & $A$ \\
\hline & Sun [51] & 0.1598 & 0.2006 & 0.1247 & 0.5149 & $\Theta$ \\
\hline & Yuan [55] & 0.7434 & 0.0668 & 0.1898 & 0 & $A$ \\
\hline & Xiao [15] & 0.7438 & 0.0790 & 0.1772 & 0 & $A$ \\
\hline & Proposed & 0.7659 & 0.0246 & 0.2096 & 0 & $A$ \\
\hline \multirow{7}{*}{1 trust paradox } & DS [24] & 0 & 1 & 0 & 0 & $B$ \\
\hline & Murphy [52] & 0.4880 & 0.0240 & 0.4880 & 0 & $A / C$ \\
\hline & Deng [53] & 0.4880 & 0.0240 & 0.4880 & 0 & $A / C$ \\
\hline & Sun $[51]$ & 0.1655 & 0.0468 & 0.1655 & 0.6221 & $\Theta$ \\
\hline & Yuan [55] & 0.4880 & 0.0240 & 0.4880 & 0 & $A / C$ \\
\hline & Xiao [15] & 0.4880 & 0.0240 & 0.4880 & 0 & $A / C$ \\
\hline & Proposed & 0.4900 & 0.0200 & 0.4900 & 0 & $A / C$ \\
\hline \multirow{7}{*}{ Highly conflicting paradox } & DS [24] & 0 & 0.9153 & 0.0847 & 0 & $B$ \\
\hline & Murphy [52] & 0.8490 & 0.1520 & 0.0101 & 0.0010 & $A$ \\
\hline & Deng [53] & 0.9499 & 0.0411 & 0.0080 & 0.0001 & $A$ \\
\hline & Sun [51] & 0.2361 & 0.1439 & 0.0513 & 0.5687 & $\Theta$ \\
\hline & Yuan [55] & 0.9709 & 0.0004 & 0.0228 & 0.0058 & $A$ \\
\hline & Xiao [15] & 0.9806 & 0.0078 & 0.0002 & 0.0114 & $A$ \\
\hline & Proposed & 0.9868 & 0.0055 & 0.0003 & 0.0075 & $A$ \\
\hline
\end{tabular}

method, Deng's method, Yuan's method, Xiao's method, and the proposed method can deal with the conflict to some extent in the 1 trust paradox. The numerical example in this paradox is an extreme case. The target proposition could be accurately identified as long as there is an increase in the pieces of evidence.

As is evident from Figure 2(d) and Table 2, the DS algorithm assigns to the wrong proposition $B$ the higher belief degree of $m(B)=0.9153$. Sun's method gives the true proposition $A$ more trust and allots a higher belief degree to $\Theta$ in the event of different amounts of evidence. Murphy's method, Deng's method, Yuan's method, Xiao's method, and the proposed method all present reasonable results and recognize the target proposition $A$ in the highly conflicting paradox.

From the above analysis, it can be concluded that the DS and Yager algorithms were not able to handle any of the paradoxes. Murphy's algorithm only averaged the evidence without considering the correlation between pieces of evidence and performed the calculations using the same weight; hence, the results were not authentic. Sun's algorithm was able to solve the paradoxes to some extent on account of having better combination rules; however, it allocated a higher belief degree to the uncertainty proposition $\Theta$. Deng's algorithm, Yuan's method, Xiao's method, and the proposed method provided reasonable results and recognized the target propositions in all four paradoxes.

The comparison in Figure 2(e) shows that the proposed method had basically the same effect with Deng's method and Yuan's method in handling the completely conflicting paradox and the 1 trust paradox; however, it was more efficient than Xiao's method in handling the 0 trust paradox and the highly conflicting paradox. The reason was that the 
TABLE 2: Fusion results of the increasing of the evidence in highly conflicting paradox.

\begin{tabular}{|c|c|c|c|c|c|}
\hline \multirow{2}{*}{ Methods } & & \multicolumn{4}{|c|}{ Propositions } \\
\hline & & $A$ & $B$ & $C$ & $\Theta$ \\
\hline \multirow{3}{*}{ DS [24] } & $m_{1} m_{2} m_{3}$ & 0 & 0.9153 & 0.0847 & 0 \\
\hline & $m_{1} m_{2} m_{3} m_{4}$ & 0 & 0.9153 & 0.0847 & 0 \\
\hline & $m_{1} m_{2} m_{3} m_{4} m_{5}$ & 0 & 0.9153 & 0.0847 & 0 \\
\hline \multirow{3}{*}{ Murphy [52] } & $m_{1} m_{2} m_{3}$ & 0.3324 & 0.5909 & 0.0540 & 0.0227 \\
\hline & $m_{1} m_{2} m_{3} m_{4}$ & 0.6170 & 0.3505 & 0.0272 & 0.0053 \\
\hline & $m_{1} m_{2} m_{3} m_{4} m_{5}$ & 0.8490 & 0.1520 & 0.0101 & 0.0010 \\
\hline \multirow{3}{*}{ Deng [53] } & $m_{1} m_{2} m_{3}$ & 0.4477 & 0.4546 & 0.0644 & 0.0333 \\
\hline & $m_{1} m_{2} m_{3} m_{4}$ & 0.8007 & 0.1640 & 0.0283 & 0.0070 \\
\hline & $m_{1} m_{2} m_{3} m_{4} m_{5}$ & 0.9499 & 0.0411 & 0.0080 & 0.0001 \\
\hline \multirow{3}{*}{ Sun [51] } & $m_{1} m_{2} m_{3}$ & 0.1326 & 0.1948 & 0.0452 & 0.6307 \\
\hline & $m_{1} m_{2} m_{3} m_{4}$ & 0.2020 & 0.1659 & 0.0506 & 0.5815 \\
\hline & $m_{1} m_{2} m_{3} m_{4} m_{5}$ & 0.2361 & 0.1439 & 0.0513 & 0.5687 \\
\hline \multirow{3}{*}{ Yuan [55] } & $m_{1} m_{2} m_{3}$ & 0.7728 & 0.0186 & 0.1128 & 0.0958 \\
\hline & $m_{1} m_{2} m_{3} m_{4}$ & 0.9101 & 0.0031 & 0.0593 & 0.0275 \\
\hline & $m_{1} m_{2} m_{3} m_{4} m_{5}$ & 0.9709 & 0.0004 & 0.0228 & 0.0058 \\
\hline \multirow{3}{*}{ Xiao [15] } & $m_{1} m_{2} m_{3}$ & 0.6239 & 0.0817 & 0.0101 & 0.2843 \\
\hline & $m_{1} m_{2} m_{3} m_{4}$ & 0.9130 & 0.0140 & 0.0017 & 0.0712 \\
\hline & $m_{1} m_{2} m_{3} m_{4} m_{5}$ & 0.9806 & 0.0078 & 0.0002 & 0.0114 \\
\hline \multirow{3}{*}{ Proposed } & $m_{1} m_{2} m_{3}$ & 0.6002 & 0.1683 & 0.0110 & 0.2205 \\
\hline & $m_{1} m_{2} m_{3} m_{4}$ & 0.9144 & 0.0355 & 0.0019 & 0.0482 \\
\hline & $m_{1} m_{2} m_{3} m_{4} m_{5}$ & 0.9868 & 0.0055 & 0.0003 & 0.0075 \\
\hline
\end{tabular}

proposed method considered both the credibility degree and the support degree of the evidence. The evidence was divided into two separate categories according to the judgment rules. The type of evidence with a high credibility degree was allocated a constant credibility weight (normalization factor 1), and another was allocated a corresponding credibility weight. This ensured that the high credibility degree evidence could play a crucial role in the final combination. Hence, the feasibility and rationality of the proposed method were successfully verified through the numerical experiments with common paradoxes. They constitute the theoretical foundation for the practical application that is described in the following section.

\section{Application and Analyses}

In order to demonstrate the effectiveness of the proposed method in a practical application, we provide a case analysis of the target decision making of the biological system.

5.1. Problem Statement. The core of the parameter control in a biological system under an intelligent multisensor network is intelligent selection and decision thereon [67]. It uses a large number of densely deployed intelligent sensor nodes to collect and analyse the data on the system, which is later effectively processed in accordance with the DS evidence theory. It also uses intelligent decision-making to replace the traditional means to improve the speed at which decisions are made and the accuracy of these decisions.

In this experiment, biogas slurry, after anaerobic fermentation, was diluted to different concentrations as a nutrient solution for a hydroponic system. It was presumed that the frame of discernment $\Theta$ consisted of six concentrations as $\Theta=\left\{C_{1}, C_{2}, C_{3}, C_{4}, C_{5}, C_{6}\right\}$. The data on the system's parameters, including the plant growth characteristics (plant height, plant weight, and root weight), the content of mineral elements $(N, P$, and $K)$ in different concentrations, and pollutant removal $\left(\mathrm{NH}_{3}-\mathrm{N}, \mathrm{COD}, \mathrm{TN}, \mathrm{TP}\right)$, were acquired through biological sensors that were positioned on the hydroponic system. Twenty data elements were collected for each group of parameters. For the analysis, we selected five groups of representative parameters for simplicity: plant height, plant weight, the content of $N$, TP removal, and COD removal.

5.2. Preprocessing of the Data from the Sensors. The data that acquired were preprocessed using the boxplot [68]. Figure 3 shows the data processing based on the boxplot. Table 3 shows the median data that were used to remove the outliers. The BPA was obtained by modeling the data based on the Hamming distance and typical samples. Table 4 shows the BPAs of the five groups of parameters of the hydroponic system. $m_{1}, m_{2}, m_{3}, m_{4}$, and $m_{5}$ denote the five groups of evidence that were input into the modified DS evidence fusion algorithm.

\subsection{Concentration Decision-Making Based on the Proposed Method}

Step 1. The conflicting factor matrix $\mathbf{K}_{5 \times 5}$ can be constructed as follows:

$$
\mathbf{K}=\left[\begin{array}{ccccc}
0 & 0.8016 & 0.8226 & 0.8245 & 0.7996 \\
0.8016 & 0 & 0.8228 & 0.8267 & 0.8037 \\
0.8226 & 0.8228 & 0 & 0.8268 & 0.8186 \\
0.8245 & 0.8267 & 0.8268 & 0 & 0.7903 \\
0.7996 & 0.8037 & 0.8186 & 0.7903 & 0
\end{array}\right]
$$

Step 2. The average conflicting factor $\bar{K}_{i}$ of the piece of evidence $\mathbf{m}_{i}$ is calculated as follows:

$$
\left[\bar{K}_{i}\right]=\left[\begin{array}{lllll}
0.8121 & 0.8137 & 0.8227 & 0.8171 & 0.8031
\end{array}\right]^{\mathrm{T}} .
$$

The average conflicting factor $\bar{K}$ of the conflicting factor matrix is calculated as follows:

$$
\bar{K}=0.8137 \text {. }
$$




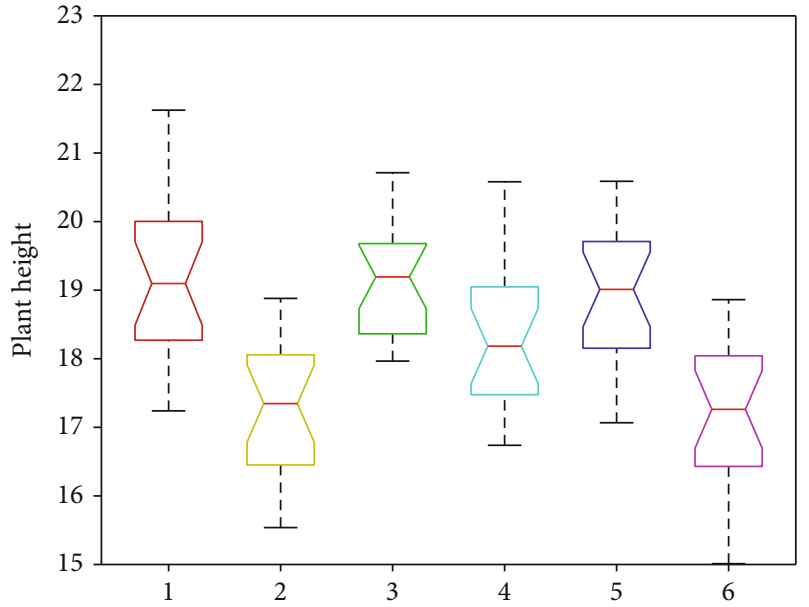

(a)

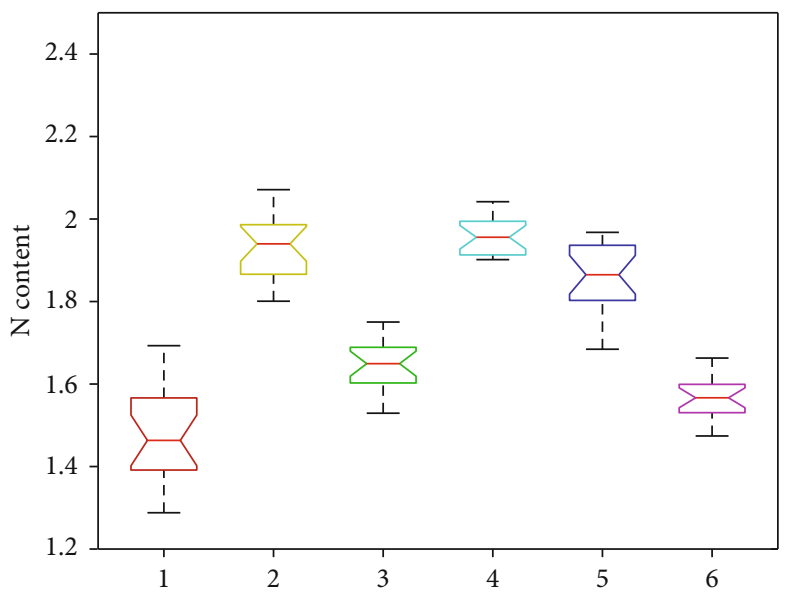

(c)

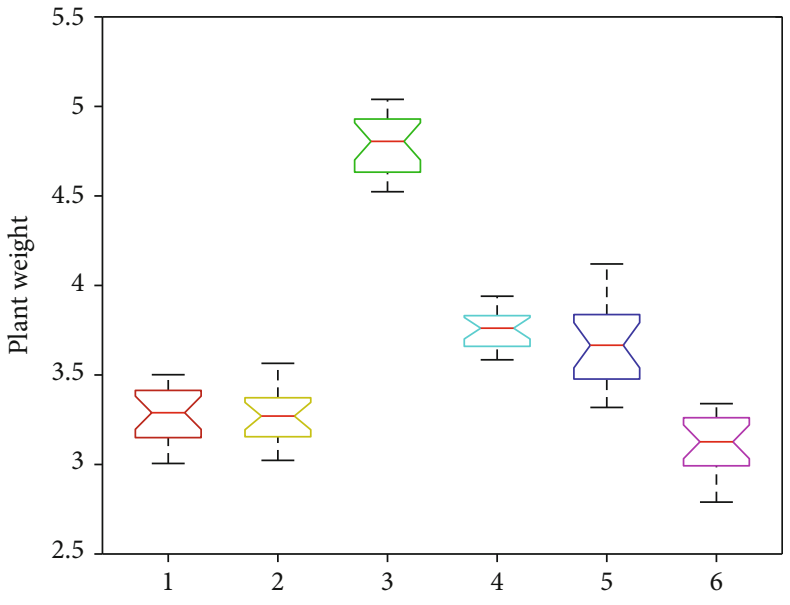

(b)

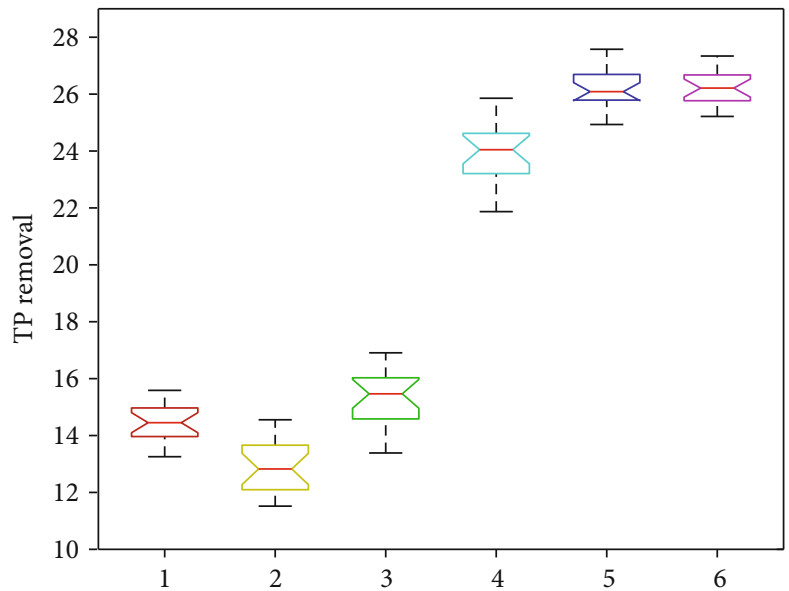

(d)

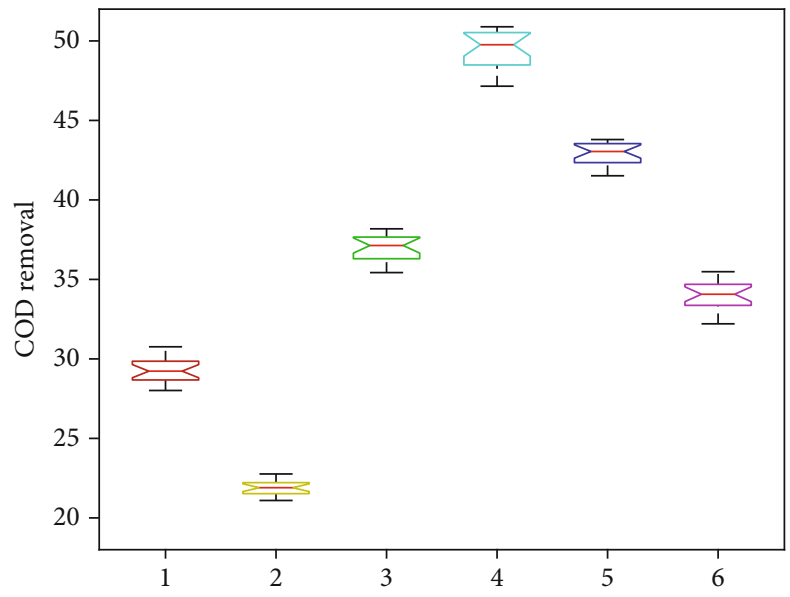

(e)

Figure 3: Data processing based on the boxplot. (a) Plant height. (b) Plant weight. (c) The content of N. (d) TP removal. (e) COD removal.

Step 3. The belief entropy matrix can be constructed as follows:

$E_{d}(\mathbf{M})=\left[\begin{array}{lllll}2.5751 & 2.5814 & 2.6253 & 2.3935 & 2.4926\end{array}\right]^{\mathrm{T}}$.
Average belief entropy $\bar{E}_{d}(\mathbf{M})$ of the evidence matrix is calculated as follows:

$$
\bar{E}_{d}(\mathbf{M})=2.5336 .
$$


TABLE 3: The median data after preprocessing.

\begin{tabular}{lcccccc}
\hline \multirow{2}{*}{ Median } & \multicolumn{7}{c}{ Concentrations } \\
& $C 1$ & $C 2$ & $C 3$ & $C 4$ & $C 5$ & $C 6$ \\
\hline Plant height $(\mathrm{cm})$ & 19.02 & 17.25 & 19.18 & 18.23 & 19.00 & 17.15 \\
Plant weight $(\mathrm{g})$ & 3.27 & 3.30 & 4.78 & 3.87 & 3.71 & 3.10 \\
Content of $N(\%)$ & 1.42 & 1.91 & 1.63 & 1.98 & 1.86 & 1.55 \\
TP removal $(\mathrm{mg} / \mathrm{L})$ & 14.47 & 13.01 & 15.32 & 24.20 & 26.05 & 26.39 \\
COD removal $(\mathrm{mg} / \mathrm{L})$ & 29.52 & 21.83 & 37.10 & 49.64 & 42.79 & 34.34 \\
\hline
\end{tabular}

TABLE 4: The BPAs of five groups of parameters of the hydroponic system.

\begin{tabular}{lccccccc}
\hline \multirow{2}{*}{ Evidence } & \multicolumn{7}{c}{ Propositions } \\
& $C 1$ & $C 2$ & $C 3$ & $C 4$ & $C 5$ & $C 6$ & $\Theta$ \\
\hline$m_{1}$ & 0.1991 & 0.0815 & 0.2458 & 0.1657 & 0.2233 & 0.0745 & 0.0101 \\
$m_{2}$ & 0.1211 & 0.1247 & 0.2790 & 0.1900 & 0.1765 & 0.1017 & 0.0070 \\
$m_{3}$ & 0.1353 & 0.1858 & 0.1568 & 0.1889 & 0.1806 & 0.1486 & 0.0040 \\
$m_{4}$ & 0.0886 & 0.0578 & 0.1100 & 0.2716 & 0.2400 & 0.2319 & 0.0001 \\
$m_{5}$ & 0.0953 & 0.0327 & 0.1906 & 0.2635 & 0.2535 & 0.1543 & 0.0101 \\
\hline
\end{tabular}

Step 4. The evidence can be divided into two categories: the high credibility degree matrix $\mathbf{M}_{h}=\left[\mathbf{m}_{5}\right]^{\mathrm{T}}$ and the general credibility degree matrix $\mathbf{M}_{g}=\left[\begin{array}{llll}\mathbf{m}_{1} & \mathbf{m}_{2} & \mathbf{m}_{3} & \mathbf{m}_{4}\end{array}\right]^{\mathrm{T}}$.

Step 5. The credibility weighted factor $C_{w i}$ after normalization can be constructed as follows:

$$
C_{w i}=\left[\begin{array}{lllll}
0.1969 & 0.1961 & 0.1907 & 0.2106 & 0.2057
\end{array}\right]^{\mathrm{T}} .
$$

Step 6. A distance measurement matrix $\mathbf{D}$ can be constructed as follows:

$$
\mathbf{D}=\left[\begin{array}{ccccc}
0 & 0.0793 & 0.1240 & 0.1838 & 0.1285 \\
0.0793 & 0 & 0.1027 & 0.1758 & 0.1246 \\
0.1240 & 0.1027 & 0 & 0.1380 & 0.1363 \\
0.1838 & 0.1758 & 0.1380 & 0 & 0.0823 \\
0.1285 & 0.1246 & 0.1363 & 0.0823 & 0
\end{array}\right] .
$$

Step 7. The similarity matrix of the evidence is derived as follows:

$$
\mathbf{S}=\mathbf{I}-\mathbf{D}=\left[\begin{array}{lllll}
1.0000 & 0.9207 & 0.8760 & 0.8162 & 0.8715 \\
0.9207 & 1.0000 & 0.8973 & 0.8242 & 0.8754 \\
0.8760 & 0.8973 & 1.0000 & 0.8620 & 0.8637 \\
0.8162 & 0.8242 & 0.8620 & 1.0000 & 0.9177 \\
0.8715 & 0.8754 & 0.8637 & 0.9177 & 1.0000
\end{array}\right] \text {. }
$$

Step 8. The support weighted factor $S_{w i}$ of the evidence is normalized as follows:

$$
S_{w i}=\left[\begin{array}{lllll}
0.1997 & 0.2016 & 0.2005 & 0.1960 & 0.2022
\end{array}\right]^{\mathrm{T}} .
$$

Step 9. The credibility weighted factor $C_{w i}$ and the support weighted factor $S_{w i}$ are integrated to form the final weight to adjust the BPAs of the evidence. The weighted evidence $\operatorname{WAE}\left(m\left(C_{j}\right)\right)$ is fused through the DS combination rule four times. Figure 4 and Table 5 show the final results of the fusion of the evidence from multiple sensors.

5.4. Analyses. Figure 3 shows the outliers based on the boxplot. The boxplot starts from the actual data and does not assume a statistical distribution, and the expressed form of the quartile is free from the influence of abnormal data. The calculation of the median data shown in Table 3 is also easily realized. As shown in Table 5, Murphy's algorithm, Deng's algorithm, Yuan's algorithm, Xiao's algorithm, and the proposed method were able to make an accurate decision on the target concentration C4. The DS algorithm and Sun's algorithm arrive at the wrong target ( $C 5$ and $\Theta$, respectively). The comparison in Figure 4(a) shows that the fusion algorithm of Murphy $(C 4=0.3521, C 5=0.3430)$ makes only a small distinction between $C 4$ and $C 5$. The target concentration was not able to be recognized once the data that were collected by the multisensor system changed even slightly. The proposed method allocates a higher belief degree to the target concentration C4. Figure 4(b) shows that the proposed method allocates the highest belief degree to the target concentration C4 (approximately 50.98\%). Hence, under these experimental conditions, the proposed method was able to decrease the uncertainty in the system to facilitate effective decision-making.

Table 6 shows the comparison of the running time of Yuan's algorithm, Xiao's algorithm, and the proposed method. Begin by creating an evidence matrix that generated randomly (we selected $500 \times 6,1000 \times 6$, and $2000 \times 6$ ). Adjust the ratio of the high credibility degree evidence in the evidence matrix, reaching $10 \%, 20 \%, 50 \%, 80 \%$, and $100 \%$, respectively. As is evident from Table 6, when the ratio reaches $10 \%$, there is a minimal effect on reducing the running time. When the ratio reaches $100 \%$, the running time can be reduced significantly. The running time of the proposed method decreases as the ratio of the high credibility degree evidence increases. This effect would be more evident with the increase of the pieces of evidence as shown in Table 6. So the calculation burden can be significantly reduced by the grouping of evidence.

The above analysis verifies the effectiveness of the proposed method for a practical application on decisionmaking regarding the target nutrient solution concentrations in a biological system. The proposed method provides higher accuracy and lower computation compared to other related methods in the practical application. 


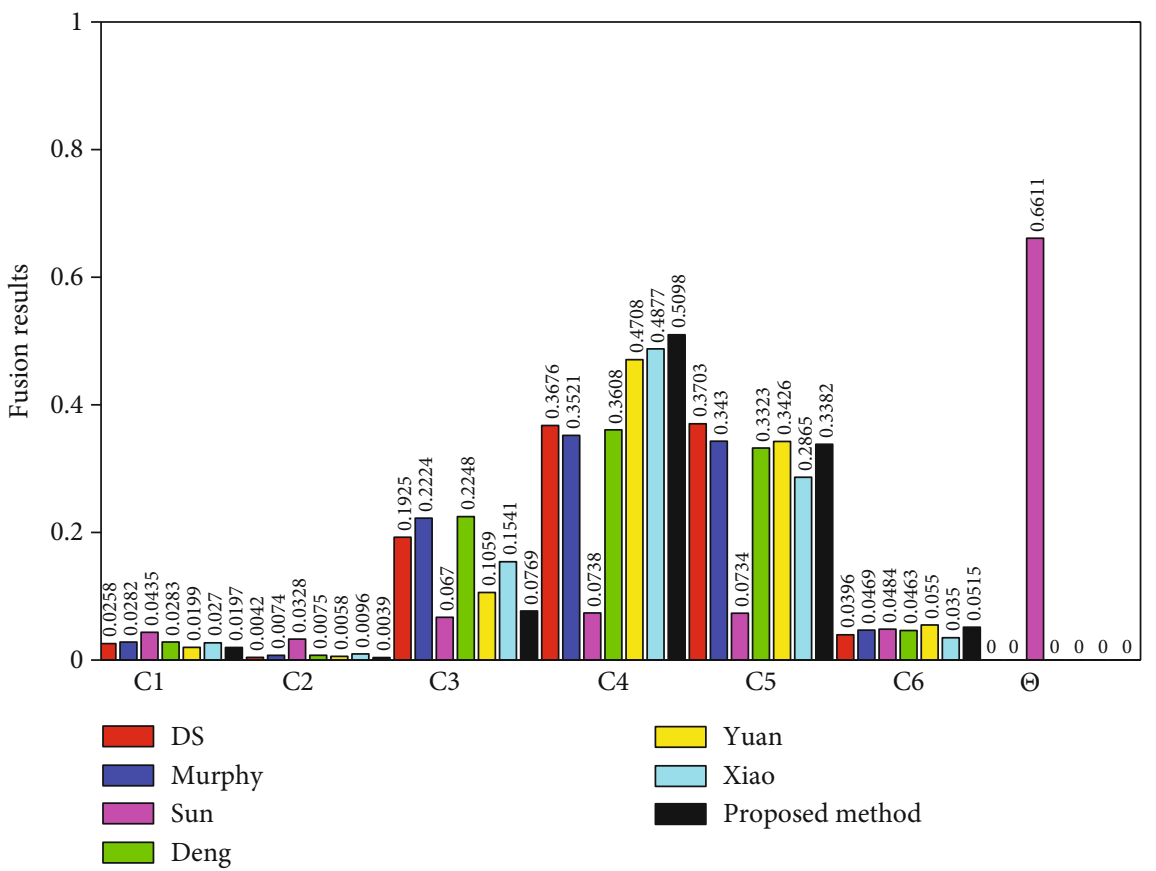

(a)

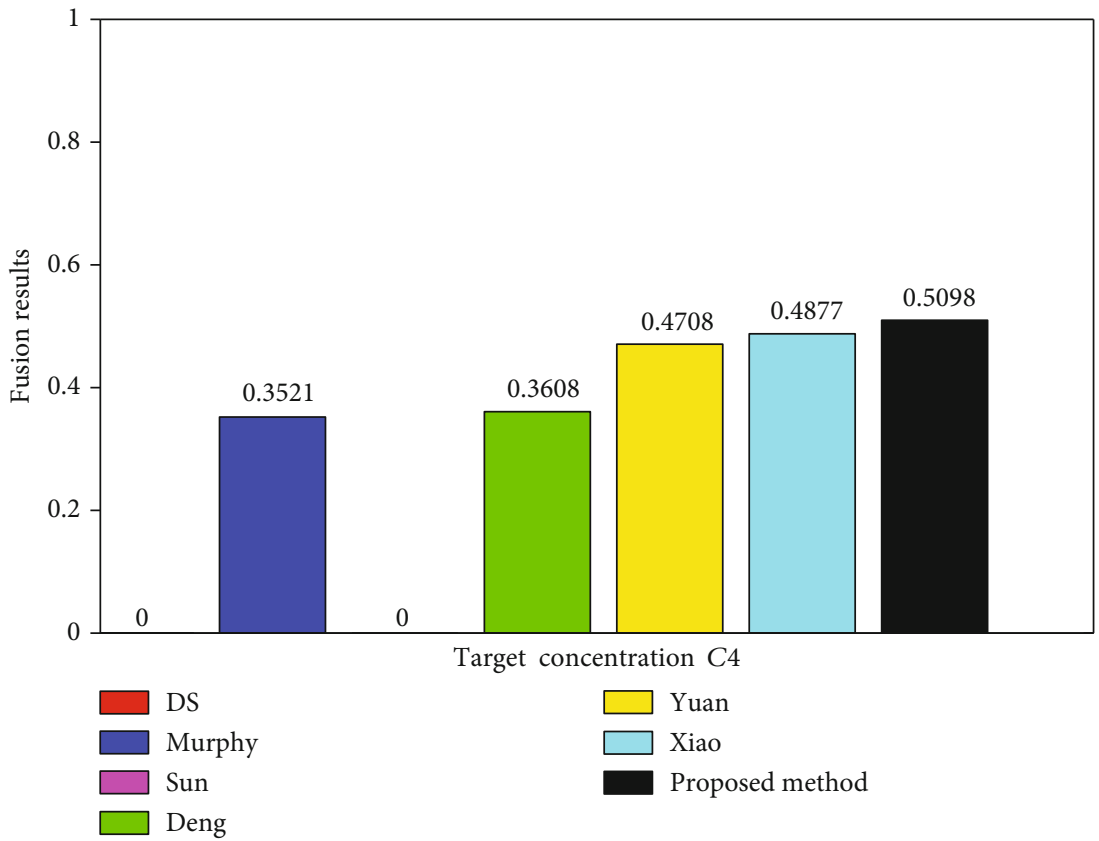

(b)

Figure 4: Fusion results in the target concentration decision-making. (a) Fusion results of the different methods. (b) Fusion results of the target concentration $\mathrm{C} 4$.

\section{Conclusions}

This paper first time proposed the concept of the grouping of evidence. Based on the concept, the proposed method was summarized in two main procedures. Firstly, the method used a combination of information entropy and the degree of conflict of the evidence as the judgment rule and divided the entire body of evidence into two separate categories: high credibility degree evidence and general credibility degree evidence. Secondly, for the grouped evidence, the method considered both the credibility weighted factors and the support weighted factors. The grouping of evidence could reduce the computational load, and the two weighted factors could improve the accuracy when fusing highly conflicting evidence.

The calculation of the credibility weighted factors was based on the Deng entropy. The appropriate credibility weighted factor was assigned to each piece of evidence. The 
TABLE 5: Fusion results in the target concentration decision-making.

\begin{tabular}{|c|c|c|c|c|c|c|c|c|}
\hline \multirow{2}{*}{ Methods } & \multicolumn{7}{|c|}{ Propositions } & \multirow{2}{*}{ Target } \\
\hline & $C 1$ & $C 2$ & $C 3$ & C4 & C5 & C6 & $\Theta$ & \\
\hline DS [24] & 0.0258 & 0.0042 & 0.1925 & 0.3676 & 0.3703 & 0.0396 & 0 & C5 \\
\hline Murphy [52] & 0.0282 & 0.0074 & 0.2224 & 0.3521 & 0.3430 & 0.0469 & 0 & $C 4$ \\
\hline Sun [51] & 0.0435 & 0.0328 & 0.0670 & 0.0738 & 0.0734 & 0.0484 & 0.6611 & $\Theta$ \\
\hline Deng [53] & 0.0283 & 0.0075 & 0.2248 & 0.3608 & 0.3323 & 0.0463 & 0 & $C 4$ \\
\hline Yuan [55] & 0.0199 & 0.0058 & 0.1059 & 0.4708 & 0.3426 & 0.0550 & 0 & $C 4$ \\
\hline Xiao [15] & 0.0270 & 0.0096 & 0.1541 & 0.4877 & 0.2865 & 0.0350 & 0 & $C 4$ \\
\hline Proposed & 0.0197 & 0.0039 & 0.0769 & 0.5098 & 0.3382 & 0.0515 & 0 & $C 4$ \\
\hline
\end{tabular}

TABLE 6: Comparison of the running time.

\begin{tabular}{|c|c|c|c|c|c|c|c|c|}
\hline & Evidence & Ratio (\%) & 0 & 10 & 20 & 50 & 80 & 100 \\
\hline \multirow{9}{*}{ Running time (s) } & \multirow{3}{*}[\mathbf{m}]{$_{500 \times 6}$} & Yuan [55] & 1.723 & 1.723 & 1.723 & 1.723 & 1.723 & 1.723 \\
\hline & & Xiao [15] & 1.633 & 1.633 & 1.633 & 1.633 & 1.633 & 1.633 \\
\hline & & Proposed & 1.770 & 1.713 & 1.668 & 1.657 & 1.618 & 1.604 \\
\hline & \multirow{3}{*}[\mathbf{m}]{$_{1000 \times 6}$} & Yuan [55] & 3.544 & 3.544 & 3.544 & 3.544 & 3.544 & 3.544 \\
\hline & & Xiao [15] & 3.329 & 3.329 & 3.329 & 3.329 & 3.329 & 3.329 \\
\hline & & Proposed & 5.908 & 4.428 & 4.273 & 2.673 & 2.176 & 1.809 \\
\hline & \multirow{3}{*}[\mathbf{m}]{$_{2000 \times 6}$} & Yuan [55] & 28.402 & 28.402 & 28.402 & 28.402 & 28.402 & 28.402 \\
\hline & & Xiao [15] & 26.108 & 26.108 & 26.108 & 26.108 & 26.108 & 26.108 \\
\hline & & Proposed & 35.179 & 26.969 & 23.531 & 17.575 & 10.476 & 7.196 \\
\hline
\end{tabular}

calculation of the support weighted factors was based on the Jousselme distance function. The two weighted factors were then integrated to arrive at the final weight to adjust the bodies of evidence before applying the DS combination rule. Numerical examples of various paradoxes and a decisionmaking case analysis on the biological system were presented to verify the rationality and effectiveness of the proposed fusion method. The simulation results and analyses indicated the following:

(1) In dealing with the paradoxes, the proposed method could provide reasonable results and recognize the target propositions in all four paradoxes. In handling the completely conflicting paradox and the 1 trust paradox, it had the same effect as Deng's algorithm, Yuan's algorithm, and Xiao's algorithm. In handling the 0 trust paradox $(m(A)=0.7659)$ and the highly conflicting paradox $(m(A)=0.9868)$, it was more accuracy than Xiao's algorithm $(m(A)=0.7438$ and $m(A)=0.9806$, respectively)

(2) In dealing with the decision-making case analysis of the biological system, the proposed method could identify the target concentration $C 4$ accurately and improved the accuracy of decision making from $48.77 \%$ to $50.98 \%$

(3) In the analysis of the running time of algorithms, the running time increased because of adding the processes of comparing and grouping when the ratio of the high credibility degree evidence was zero. Once the ratio increased, the running time would be reduced accordingly. Thus, the running time of the proposed method decreased as the ratio of the high credibility degree evidence increased. This effect would be more obvious with the increase of the pieces of evidence

From the above discussion, the proposed method was able to effectively diminish conflicts as well as arrive at a precise decision and significantly reduce the calculation burden. The proposed method was found to be more efficient and precise as compared with the other related methods.

In later studies, we intend to develop a generalized judgment rule of the grouping of evidence to make it more applicable and efficient to fit the practical applications. Meanwhile, with the increasing number of multisensors in intelligent systems, more effective solutions should be researched according to the huge computation burden for the system.

\section{Data Availability}

The data used to support the findings of this study are available from the corresponding author upon request.

\section{Conflicts of Interest}

The authors declare that there is no conflict of interest regarding the publication of this paper. 


\section{Acknowledgments}

The authors wish to acknowledge the financial support of the National Natural Science Foundation of China under Projects 61471224 and 61801270 and Shandong Key Research and Development Program under Grant 2018GHY115022.

\section{References}

[1] X. Xu, F. Zhang, G. Yang, L. Xie, Q. Liu, and B. Li, “Throughput-guaranteed distributed channel assignment and scheduling algorithms with low complexity for multichannel wireless sensor networks," Journal of Sensors, vol. 2019, Article ID 6171625, 12 pages, 2019.

[2] J. Alcaina, Á. Cuenca, J. Salt, M. Zheng, and M. Tomizuka, "Energy-efficient control for an unmanned ground vehicle in a wireless sensor network," Journal of Sensors, vol. 2019, Article ID 7085915, 16 pages, 2019.

[3] W. Kim, H. W. Moon, and Y. J. Yoon, "Adaptive triangular deployment of underwater wireless acoustic sensor network considering the underwater environment," Journal of Sensors, vol. 2019, Article ID 6941907, 11 pages, 2019.

[4] Y. Li, X. Wang, and D. Liu, "3D autonomous navigation line extraction for field roads based on binocular vision," Journal of Sensors, vol. 2019, Article ID 6832109, 16 pages, 2019.

[5] H. Wang, X. Lou, Y. Cai, Y. Li, and L. Chen, "Real-time vehicle detection algorithm based on vision and lidar point cloud fusion," Journal of Sensors, vol. 2019, Article ID 8473980, 9 pages, 2019.

[6] X. Bai, Z. Wang, L. Sheng, and Z. Wang, "Reliable data fusion of hierarchical wireless sensor networks with asynchronous measurement for greenhouse monitoring," IEEE Transactions on Control Systems Technology, vol. 27, no. 3, pp. 1036-1046, 2019.

[7] X. Bai, Z. Wang, L. Zou, and F. E. Alsaadi, "Collaborative fusion estimation over wireless sensor networks for monitoring $\mathrm{CO}_{2}$ concentration in a greenhouse," Information Fusion, vol. 42, pp. 119-126, 2018.

[8] D. L. Hall and S. A. H. Mcmullen, Mathematical techniques in multisensor data fusion, Artech House, Boston, London, 1992, http://engr.wisc.edu.

[9] C. Harris, X. Hong, and Q. Gan, "Multisensor data fusion using Kalman filters based on neurofuzzy linearisation," in Adaptive Modelling, Estimation and Fusion from Data, Advanced Information Processing, pp. 255-280, Springer, Berlin, Heidelberg, 2002.

[10] P. A. Samara, G. N. Fouskitakis, J. S. Sakellariou, and S. D. Fassois, "A statistical method for the detection of sensor abrupt faults in aircraft control systems," IEEE Transactions on Control Systems Technology, vol. 16, no. 4, pp. 789-798, 2008.

[11] Z. J. Zhou, C. H. Hu, D. L. Xu, J. B. Yang, and D. H. Zhou, "Bayesian reasoning approach based recursive algorithm for online updating belief rule based expert system of pipeline leak detection," Expert Systems with Applications, vol. 38, no. 4, pp. 3937-3943, 2011.

[12] S. A. Sloman, "The empirical case for two systems of reasoning," Psychological Bulletin, vol. 119, no. 1, pp. 3-22, 1996.

[13] M. Truchon, "Borda and the maximum likelihood approach to vote aggregation,” Mathematical Social Sciences, vol. 55, no. 1, pp. 96-102, 2008.
[14] Z. Y. Gao, R. G. Dong, J. M. Gao, and R. X. Wang, "A method to generate basic belief assignment based on clustering analysis and its application," Journal of Xi'an Jiaotong University, vol. 50, no. 10, pp. 8-14, 2016.

[15] F. Xiao, "Multi-sensor data fusion based on the belief divergence measure of evidences and the belief entropy," Information Fusion, vol. 46, pp. 23-32, 2019.

[16] B. Kang, Y. Deng, K. Hewage, and R. Sadiq, "A method of measuring uncertainty for z-number," IEEE Transactions on Fuzzy Systems, vol. 27, no. 4, pp. 731-738, 2019.

[17] Y. Tang, D. Zhou, S. Xu, and Z. He, "A weighted belief entropy-based uncertainty measure for multi-sensor data fusion," Sensors, vol. 17, no. 4, p. 928, 2017.

[18] S. H. Oh, "Improving the error backpropagation algorithm with a modified error function," IEEE Transactions on Neural Networks, vol. 8, no. 3, pp. 799-803, 1997.

[19] B. Walczak and D. L. Massart, "Rough sets theory," Chemometrics and Intelligent Laboratory Systems, vol. 47, no. 1, pp. 1-16, 1999.

[20] L. Shen, F. E. H. Tay, L. Qu, and Y. Shen, "Fault diagnosis using rough sets theory," Computers in Industry, vol. 43, no. 1, pp. 61-72, 2000.

[21] L. A. Zadeh, "Fuzzy sets," Information and Control, vol. 8, no. 3, pp. 338-353, 1965.

[22] F. Xiao and W. Ding, "Divergence measure of pythagorean fuzzy sets and its application in medical diagnosis," Applied Soft Computing, vol. 79, pp. 254-267, 2019.

[23] L. Fei, H. Wang, L. Chen, and Y. Deng, "A new vector valued similarity measure for intuitionistic fuzzy sets based on OWA operators," Iranian Journal of Fuzzy Systems, vol. 16, no. 3, pp. 113-126, 2019.

[24] A. P. Dempster, "Upper and lower probabilities induced by a multivalued mapping," The Annals of Mathematical Statistics, vol. 38, no. 2, pp. 325-339, 1967.

[25] G. Shafer and P. Diaconis, "A mathematical theory of evidence," Journal of the American Statistical Association, vol. 73, no. 363, pp. 677-678, 1978.

[26] S. Xu, W. Jiang, X. Deng, and Y. Shou, "A modified Physaruminspired model for the user equilibrium traffic assignment problem," Applied Mathematical Modelling, vol. 55, pp. 340353,2018

[27] Q. Zhang, M. Li, and Y. Deng, "Measure the structure similarity of nodes in complex networks based on relative entropy," Physica A: Statistical Mechanics and its Applications, vol. 491, pp. 749-763, 2018.

[28] X. Zhou, Y. Hu, Y. Deng, F. T. S. Chan, and A. Ishizaka, “A dematel-based completion method for incomplete pairwise comparison matrix in AHP," Annals of Operations Research, vol. 271, no. 2, pp. 1045-1066, 2018.

[29] C. Fu, D. L. Xu, and S. L. Yang, "Distributed preference relations for multiple attribute decision analysis," Journal of the Operational Research Society, vol. 67, no. 3, pp. 457-473, 2017.

[30] W. Jiang, B. Wei, X. Liu, X. Li, and H. Zheng, "Intuitionistic fuzzy power aggregation operator based on entropy and its application in decision making," International Journal of Intelligent Systems, vol. 33, no. 1, pp. 49-67, 2018.

[31] F. Ye, J. Chen, Y. Li, and J. Kang, "Decision-making algorithm for multisensor fusion based on grey relation and DS evidence theory," Journal of Sensors, vol. 2016, Article ID 3954573, 11 pages, 2016. 
[32] F. Xiao, "EFMCDM: evidential fuzzy multicriteria decision making based on belief entropy," IEEE Transactions on Fuzzy Systems, p. 1, 2019.

[33] L. Fei, J. Xia, Y. Feng, and L. Liu, "An electre-based multiple criteria decision making method for supplier selection using Dempster-Shafer theory," IEEE Access, vol. 7, pp. 8470184716, 2019.

[34] Y. Li, J. Chen, F. Ye, and D. Liu, "The improvement of DS evidence theory and its application in IR/MMW target recognition," Journal of Sensors, vol. 2016, Article ID 1903792, 15 pages, 2016.

[35] J. Ma, W. Liu, P. Miller, and H. Zhou, “An evidential fusion approach for gender profiling," Information Sciences, vol. 333, no. 3, pp. 10-20, 2016.

[36] Z. Liu, Q. Pan, J. Dezert, and A. Martin, "Adaptive imputation of missing values for incomplete pattern classification," Pattern Recognition, vol. 52, pp. 85-95, 2016.

[37] P. Dutta, "Uncertainty Modeling in Risk Assessment Based on Dempster-Shafer Theory of Evidence with Generalized Fuzzy Focal Elements," Fuzzy Information and Engineering, vol. 7, no. 1, pp. 15-30, 2015.

[38] L. Zhang, L. Ding, X. Wu, and M. J. Skibniewski, "An improved Dempster-Shafer approach to construction safety risk perception," Knowledge-Based Systems, vol. 132, pp. 3046, 2017.

[39] X. Zheng and Y. Deng, "Dependence assessment in human reliability analysis based on evidence credibility decay model and IOWA operator," Annals of Nuclear Energy, vol. 112, pp. 673-684, 2018.

[40] T. Liu, Y. Deng, and F. Chan, "Evidential supplier selection based on dematel and game theory," International Journal of Fuzzy Systems, vol. 20, no. 4, pp. 1321-1333, 2018.

[41] F. Sabahi, "A novel generalized belief structure comprising unprecisiated uncertainty applied to aphasia diagnosis," Journal of Biomedical Informatics, vol. 62, pp. 66-77, 2016.

[42] X. Yao, S. Li, and J. Hu, "Improving rolling bearing fault diagnosis by DS evidence theory based fusion model," Journal of Sensors, vol. 2017, Article ID 6737295, 14 pages, 2017.

[43] K. Yuan, F. Xiao, L. Fei, B. Kang, and Y. Deng, "Modeling sensor reliability in fault diagnosis based on evidence theory," Sensors, vol. 16, no. 1, p. 113, 2016.

[44] F. Xiao, "A novel evidence theory and fuzzy preference approach-based multi-sensor data fusion technique for fault diagnosis," Sensors, vol. 17, no. 11, article 2504, 2017.

[45] S. Jia, K. Wang, X. Li, and T. Xu, "A novel improved probability-guided RANSAC algorithm for robot 3D map building," Journal of Sensors, vol. 2016, Article ID 3243842, 18 pages, 2016.

[46] B. C. Chen, X. Tao, M. R. Yang, C. Yu, W. M. Pan, and V. C. M. Leung, "A saliency map fusion method based on weighted DS evidence theory," IEEE Access, vol. 6, pp. 27346-27355, 2018.

[47] L. A. Zadeh, "A simple view of the Dempster-Shafer theory of evidence and its implication for the rule of combination," $A I$ Magazine, vol. 7, no. 2, pp. 85-90, 1986.

[48] P. Smets, "The combination of evidence in the transferable belief model," IEEE Transactions on Pattern Analysis and Machine Intelligence, vol. 12, no. 5, pp. 447-458, 1990.

[49] D. Dubois and H. Prade, "Representation and combination of uncertainty with belief functions and possibility measures," Computational Intelligence, vol. 4, no. 3, pp. 244-264, 1988
[50] R. R. Yager, "On the dempster-shafer framework and new combination rules," Information Sciences, vol. 41, no. 2, pp. 93-137, 1987.

[51] Q. Sun, X. Q. Ye, and W. K. Gu, "Distributed preference relations for multiple attribute decision analysis," Chinese Journal of Electronics, vol. 28, no. 8, pp. 117-119, 2000.

[52] C. K. Murphy, "Combining belief functions when evidence conflicts," Decision Support Systems, vol. 29, no. 1, pp. 1-9, 2000.

[53] D. Yong, S. WenKang, Z. ZhenFu, and L. Qi, "Combining belief functions based on distance of evidence," Decision Support Systems, vol. 38, no. 3, pp. 489-493, 2004.

[54] Z. Zhang, T. Liu, D. Chen, and W. Zhang, "Novel algorithm for identifying and fusing conflicting data in wireless sensor networks," Sensors, vol. 14, no. 6, pp. 9562-9581, 2014.

[55] K. Yuan, F. Xiao, L. Fei, B. Kang, and Y. Deng, "Conflict management based on belief function entropy in sensor fusion," Springerplus, vol. 5, no. 1, p. 638, 2016.

[56] R. Jiroušek and P. P. Shenoy, "A new definition of entropy of belief functions in the Dempster-Shafer theory," International Journal of Approximate Reasoning, vol. 92, pp. 49-65, 2018.

[57] F. Ye, J. Chen, and Y. Tian, "A Robust DS Combination Method Based on Evidence Correction and Conflict Redistribution," Journal of Sensors, vol. 2018, Article ID 6526018, 12 pages, 2018.

[58] M. N. Khan and S. Anwar, "Paradox elimination in DempsterShafer combination rule with novel entropy function: application in decision-level multi-sensor fusion," Sensors, vol. 19, no. 21, article 4810, 2019.

[59] M. Ma and J. An, "Combination of evidence with different weighting factors: a novel probabilistic-based dissimilarity measure approach," Journal of Sensors, vol. 2015, Article ID 509385, 9 pages, 2015.

[60] H. Jiang, R. Wang, Z. Gao, J. Gao, and H. Wang, "Classification of weld defects based on the analytical hierarchy process and Dempster-Shafer evidence theory," Journal of Intelligent Manufacturing, vol. 30, no. 4, pp. 2013-2024, 2019.

[61] A. L. Jousselme, D. Grenier, and É. Bossé, "A new distance between two bodies of evidence," Information Fusion, vol. 2, no. 2, pp. 91-101, 2001.

[62] A. L. Jousselme and P. Maupin, "Distances in evidence theory: comprehensive survey and generalizations," International Journal of Approximate Reasoning, vol. 53, no. 2, pp. 118$145,2012$.

[63] Y. Deng, "Deng entropy," Chaos, Solitons \& Fractals, vol. 91, no. 3, pp. 549-553, 2016.

[64] C. E. Shannon, "A mathematical theory of communication," ACM SIGMOBILE Mobile Computing and Communications Review, vol. 5, no. 1, pp. 3-55, 2001.

[65] R. R. Yager, "Entropy and specificity in a mathematical theory of evidence," International Journal of General Systems, vol. 9, no. 4, pp. 249-260, 1983.

[66] X. Z. Guo and X. L. Xin, "Partial entropy and relative entropy of fuzzy sets," Fuzzy System \& Mathematics, vol. 19, no. 2, pp. 97-102, 2005.

[67] S. X. Zhang, J. H. Lin, L. Su, and Z. P. Zhou, "Optical gain and laser characteristics of InGaN quantum wells on ternary InGaN substrates," Analytical Biochemistry, vol. 564, pp. 54-63, 2019.

[68] M. L. Walker, Y. H. Dovoedo, S. Chakraborti, and C. W. Hilton, "An improved boxplot for univariate data," American Statistician, vol. 72, no. 4, pp. 348-353, 2018. 


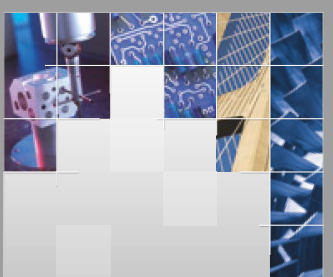

\section{Enfincering}
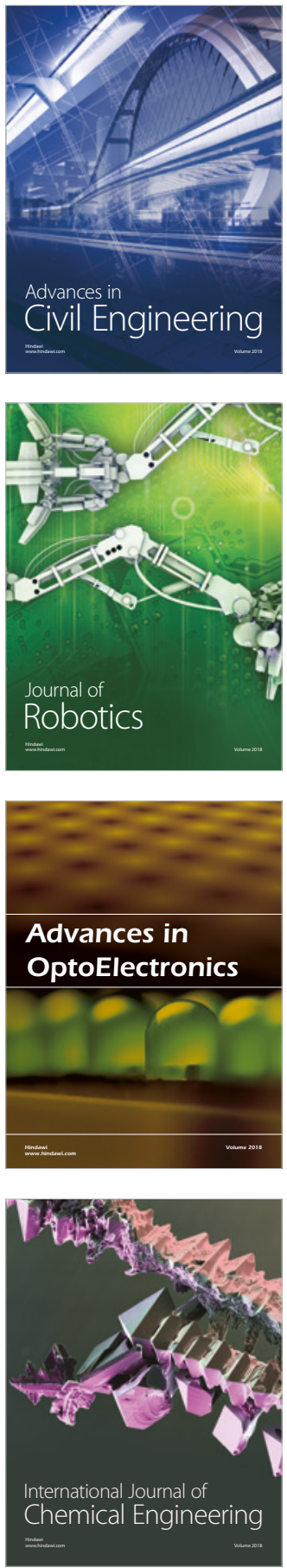

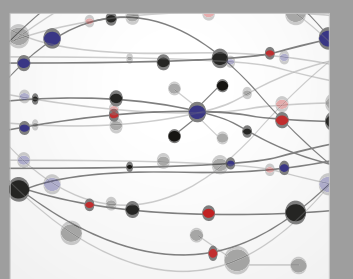

\section{Rotating \\ Machinery}

The Scientific World Journal

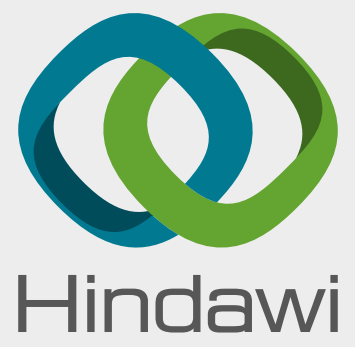

Submit your manuscripts at

www.hindawi.com
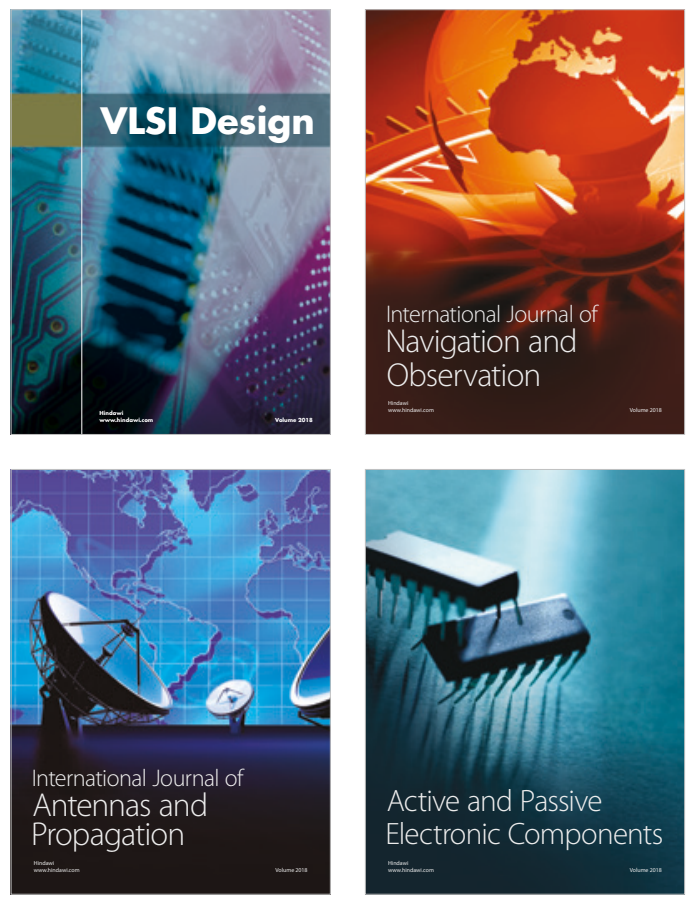
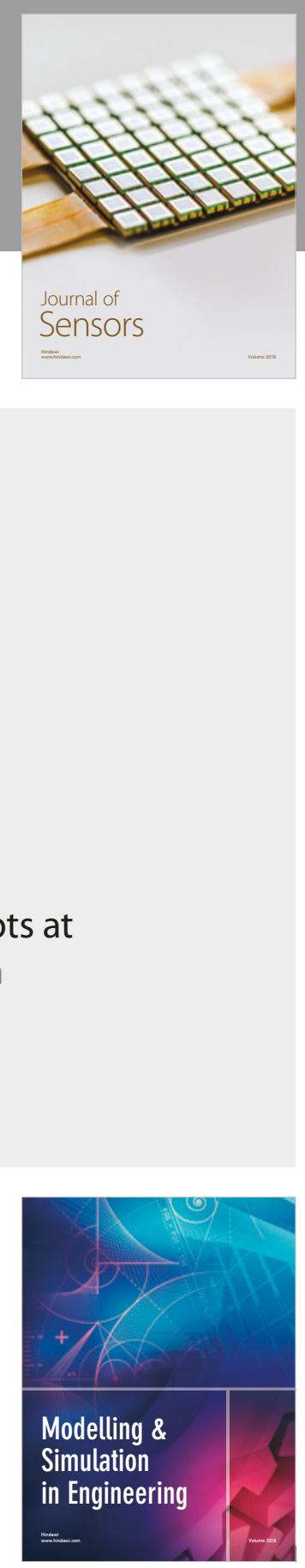

\section{Advances \\ Multimedia}
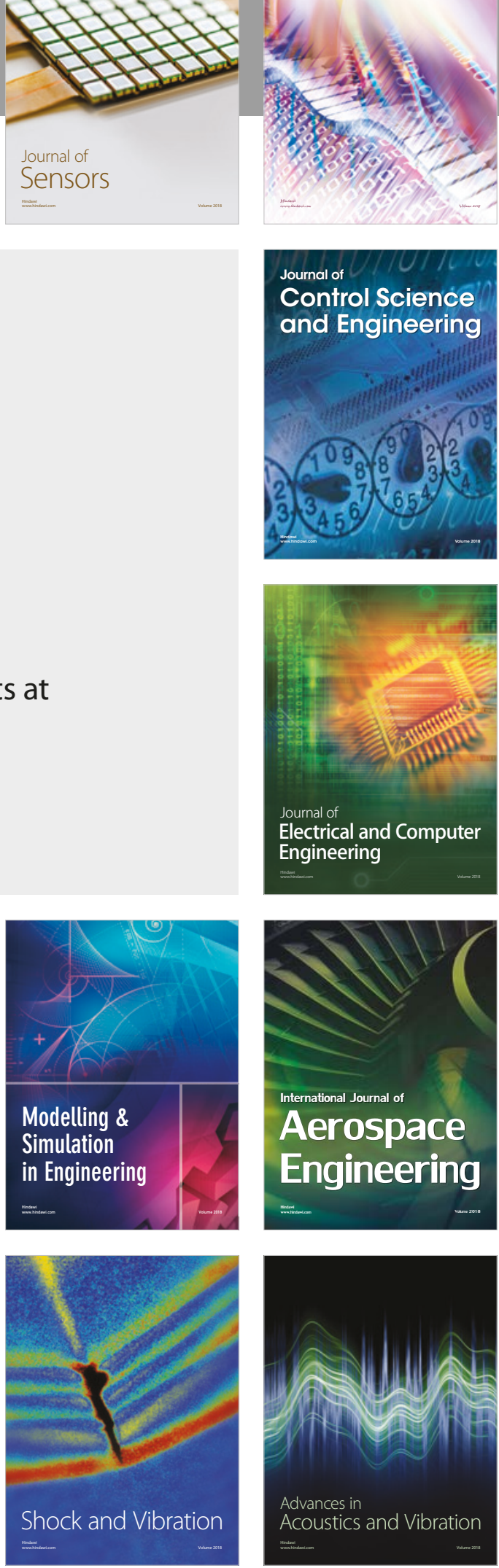